J. Dorfmeister

Nagoya Math. J.

Vol. 86 (1982), 39-83

\title{
HOMOGENEOUS SIEGEL DOMAINS
}

\author{
JOSEF DORFMEISTER
}

In 1935 E. Cartan classified all symmetric bounded domains [6]. At that time he proved that a bounded symmetric domain is homogeneous with respect to its group of holomorphic automorphisms. Thus the more general problem of investigating homogeneous bounded domains arose. It was known to E. Cartan that all homogeneous bounded domains of dimension $\leq 3$ are symmetric [6]. For domains of higher dimension little was known. The first example of a 4-dimensional, homogeneous, nonsymmetric bounded domain was provided by I. Piatetsky-Shapiro [41]. In several papers he investigated homogeneous bounded domains [20], [21], [41], [42], [43]. One of the main results is that all such domains have an unbounded realization of a certain type, as a so-called Siegel domain. But many questions still remained open. Amongst them the question for the structure and explicit form of the infinitesimal automorphisms of a homogeneous Siegel domain.

Important contributions on this problem have been provided by $\mathrm{N}$. Tanaka [54] and W. Kaup, Y. Matsushima, T. Ochiai [29]. They used graduations of the Lie algebra $g$ of infinitesimal automorphisms of a Siegel domain $D$. Starting from these two papers several articles have been published which dealt with a more precise description of the elements of g [37], special cases and examples [49], [50], [51], [56], [57], symmetric subspaces [38], the representation of $D$ as a Siegel domain of type III [39], as well as other questions.

In this work we present a new method for the description of homogeneous Siegel domains. This method entails a classification of the domains considered and makes it possible to answer several of the open question. We are able to reproduce the known results and, in some cases,

Received April 23, 1979.

This article coincides up to minor changes with chapter I of the authors "Habilitationsschrift" [13] which has been accepted by the "Mathematisch-Naturwissenschaftliche Fakultät der Westfälischen Wilhelms-Universität zu Münster”. 
get a geometric interpretation.

There are several applications of the results of this paper. We would like to mention three of them. Using the theory of representations of Jordan algebras [24] and the theory of clifford algebras [2], [8] it is possible to find an explicite description of all quasisymmetric Siegel domains. This in particular reproduces results of I. Satake [46], [47], [48], and M. Takeuchi [53]. Moreover, a Cayley transformation for quasisymmetric Siegel domains and the symmetries of a symmetric Siegel domain can be stated explicitly. Further, by the results of [10] and of this paper it is relatively easy to construct all homogeneous Siegel domains of dimension $\leq 10$. This has been previously done by S. Kaneyuki and T. Tsuji using different methods [27], [55]. Finally, it is possible to find explicit expressions for the 1-parameter groups of vector fields $X \in \mathfrak{g}_{\lambda}$. [62]

We note that for simplicity of presentation we shall concentrate on the investigation of homogeneous Siegel domains, but the method which we present can be applied as well to non-homogeneous Siegel domains. In particular this applies to investigations on non-affine infinitesimal automorphisms.

We briefly review the contents of this article. For ease in reading this paper most of the definitions and results concerning homogeneous Siegel domains and their infinitesimal automorphisms will occur in $\S 1$. In $\S 2$ we provide the tools which are characteristic for our method. In particular we define-starting from the Bergmann kernel of a Siegel domain $D(K, S)$ in $V^{c} \times U-a$ commutative algebra $\mathfrak{A}$ and a linear map $\varphi: \mathfrak{U} \rightarrow \operatorname{End}_{C} U$. In $\S 2,6$ we establish three different descriptions of homogeneous Siegel domains. Using Theorem 2.14 and [15], 8 we thus get an algebraic classification of all homogeneous Siegel domains. The characterization of these domains by morphisms of homogeneous cones is especially useful for developping examples.

As remarked above, in $\S 1$ we assemble results on the infinitesimal automorphisms $\mathrm{g}=\mathrm{g}(K, S)$ of $D(K, S)$. We there introduce the graduation $\mathfrak{g}=\mathfrak{g}_{-1}+\mathfrak{g}_{-\frac{1}{2}}+\mathfrak{g}_{0}+\mathfrak{g}_{\frac{1}{2}}+\mathfrak{g}_{1}$ of $\mathfrak{g}$. Sections 3,4 and 5 provide an explicit description of the elements of $\mathfrak{g}_{\lambda}, \lambda=0, \frac{1}{2}, 1$ for arbitrary homogeneous Siegel domains. To be more precise, in $\S 3$ we use the results of [15] on $J$-morphisms of homogeneous cones to explicitly describe $g_{0}$ and thus to clarify its structure. We use this result to investigate $\operatorname{Ker} \varphi$ and nondegenerate Siegel domains. The elements of $g_{\frac{1}{2}}$ are characterized in $\S 4$. It 
turns out that there exists a complex vector space $P_{\frac{1}{2}}$ which parametrizes $g_{2}$. In $\S 5$ we investigate $g_{1}$. We prove that $g_{1}$ is parametrized by a subspace $P_{1}$ of $\mathfrak{A}$. Further, the relations between $P_{1}$ and $P_{\frac{1}{2}}$ are clarified. We thus find a simple and unifying explanation for several results of the literature on $\mathfrak{g}_{\frac{1}{2}}$ and $\mathfrak{g}_{1}$.

We would like to mention that for the case of a symmetric Siegel domain the infinitesimal automorphisms have been described independently by I. Satake [48].

In $\S 6$ we collect the identities which arise when we express the elements of $\left[\mathfrak{g}_{\lambda}, \mathfrak{g}_{\mu}\right]$ by the parameters of the elements of $\mathfrak{g}_{\lambda}$ and $\mathfrak{g}_{\mu}$. The last section, $\S 7$, is devoted to the investigation of the radical $\mathfrak{w}$ of $g$ and provides a Levi decomposition $\mathfrak{g}=\mathfrak{l}+\mathfrak{h}+\mathfrak{w}$ of $\mathfrak{g}$. The Lie algebra $\mathfrak{l}$ of the semisimple summand $\mathfrak{l}+\mathfrak{h}$ of this Levi decomposition is the Lie algebra of infinitesimal automorphisms of a symmetric Siegel domain. The existence of such a Levi decomposition has been proved in [38]. Here we choose $\mathfrak{l}$ so that in $P_{1}^{c} \times P_{\frac{1}{2}}$ we get a canonical realization of the corresponding symmetric domain $D(\mathfrak{l})$.

Part of this work has been done during a stay at the Institute for Advanced Study, Princeton, N. J. I would like to express my thanks to A. Borel for giving me the opportunity to work at the Institute. Special thanks are due to M. Koecher who supported the author in all regards.

\section{$\S 1$. Definitions and fundamental results}

1. Let $V$ be a finite dimensional vector space over the reals $R$. We provide $V$ with the canonical topology. By $V^{c}$ we denote the complexification of $V$; the elements of $V^{c}$ will be written $z=x+i y, x, y \in V$. Real part $\operatorname{Re}(z)$, imaginary part $\operatorname{Im}(z)$ and conjugation $z \mapsto \bar{z}$ (of $V^{C}$ with respect to $V$ ) are defined as usual.

A subset $K$ of $V$ is called a regular cone (in $V$ ) provided

$K$ is open and not empty,

$$
x \in K, \alpha>0 \text { implies } \alpha x \in K,
$$$$
x, y \in K \text { implies } x+y \in K \text {, }
$$

$K$ does not contain any straight line.

We set 


$$
\text { Aut } K=\left\{W \in \mathrm{Gl}_{R} V ; W K=K\right\} \text {. }
$$

Further, for a positive definite symmetric bilinear form $\tau$ on $V$ we define the $\tau$-dual cone $K^{\tau}$ by

(1.6) $K^{\tau}=\{y \in V ; \tau(x, y)>0$ for all $x \neq 0$ in the closure of $K$ in $V\}$.

Finally, for $K$ and $\tau$ as above we set

$$
\iota(K ; x)=\int_{K \tau} e^{-\tau(x, y)} d y, \quad x \in K,
$$

where $d y$ denotes Lebesgue measure on $V$.

The function $\iota(K ;)$ on $K$ is called the invariant (of $K$ ). It satisfies the transformation formula

$$
\iota(K ; W x)=|\operatorname{det} W|^{-1} \iota(K ; x), \quad x \in K, \quad W \in \text { Aut } K .
$$

For a more detailed description of regular cones and their respective invariants we refer the reader to [17].

2. Let $K$ be a regular cone in $V$ and $U$ a finite dimensional vector space over $\boldsymbol{C}$.

A map $S: U \times U \rightarrow V^{c}$ is called a $K$-hermitian form, if

$$
S \text { is } C \text {-linear in the first argument, }
$$$$
S(u, w)=\overline{S(w, u)} \text { for all } u, w \in U,
$$$$
S(u, u) \text { lies in the closure of } K \text { (in } V \text { ), }
$$

$$
S(u, u)=0 \text { if and only if } u=0 \text {. }
$$

For a regular cone $K$ and a $K$-hermitian map $S$ we define

$$
D(K, S)=\left\{(z, u) \in V^{c} \times U ; \operatorname{Im}(z)-S(u, u) \in K\right\} .
$$

It is easily seen that $D(K, S)$ is a domain in $V^{c} \times U$.

A domain of this type is called a Siegel domain.

If $U=0$ then $D(K, S)$ only depends on $K$. Such a domain is called a tube domain and is denoted by $D(K)$,

$$
D(K)=V+i K
$$

It is immediate that $D(K) \subset D(K, S)$.

3. For a Siegel domain $D(K, S)$ in $V^{c} \times U$ we set 
(1.15) Aut $D(K, S)=\{f: D(K, S) \rightarrow D(K, S)$ biholomorph $\}$.

(1.16) Aff $D(K, S)=\left\{f \in\right.$ Aut $D(K, S), f$ affine transformation of $\left.V^{c} \times U\right\}$.

(1.17) Gl $D(K, S)=$ Aut $D(K, S) \cap\left(\mathrm{Gl}_{R} V \times G L_{C} U\right)$.

A Siegel domain $D(K, S)$ is homogeneous, if Aut $D(K, S)$ operates transitively on $D(K, S)$.

It is known [29] that Aut $D(K, S)$ operates transitively on $D(K, S)$ if and only if Aff $D(K, S)$ operates transitively on $D(K, S)$.

As standard references for results on the Lie groups Aut $D(K, S)$, Aff $D(K, S)$ and $\mathrm{Gl} D(K, S)$ we refer to [26] and [36].

4. Let $D(K, S)$ be a Siegel domain and $\Gamma \subset$ Aut $K$ a closed subgroup of Aut $K$. We call $(\Gamma, S)$ admissible, if

$\Gamma$ operates transitively on $K$,

For all $W \in \Gamma$ there exists a $\hat{W} \in \mathrm{Gl}_{c} U$ such that $(W, \hat{W}) \in \mathrm{Gl} D(K, S)$.

The following lemma shows that the assumption that $\Gamma$ is closed is inessential.

Lemma 1.1. Let $D(K, S)$ be a Siegel domain.

a) If $\omega:=\left(\left(W_{n}, \hat{W}_{n}\right) ; n \in N\right)$ is a sequence in $\mathrm{Gl} D(K, S)$ such that the sequence $\left(W_{n} ; n \in N\right)$ converges to a $W \in$ Aut $K$, then $\omega$ contains a subsequence which converges in Gl $D(K, S)$.

b) Let $\Gamma$ a (not necessarily closed) subgroup of Aut $K$ which satisfies (1.19). Then the closure $\bar{\Gamma}$ of $\Gamma$ in Aut $K$ also satisfies (1.19).

Proof. a) We choose a norm $|\cdot|$ on $U$ and a norm $\|\cdot\|$ on $V$. The norm induced on $\operatorname{End}_{C} U$ (resp. $\operatorname{End}_{\boldsymbol{R}} V$ ) will be denoted by the same letter. We prove that the sequence $\left(\hat{W}_{n} ; n \in N\right)$ is bounded in the norm $|\cdot|$ of $\operatorname{End}_{C} U$. Suppose the contrary, then we may assume that there exists a sequence $\left(x_{n} ; n \in N\right)$ in $U$ with $\left|x_{n}\right|=1, n \in N$, but $\left|\hat{W}_{n} x_{n}\right| \rightarrow \infty$. We put $y_{n}:=\left|\hat{W}_{n} x_{n}\right|^{-1} \hat{W}_{n} x_{n}$ and consider the function $u \mapsto\|S(u, u)\|$ on the unit ball of $U$. Because of (1.12) this function attains a positive minimum $d$ and maximum $e$. It then follows that

$$
\begin{aligned}
0<d & \leq\left\|S\left(y_{n}, y_{n}\right)\right\|=\left|\hat{W}_{n} x_{n}\right|^{-2}\left\|S\left(\hat{W}_{n} x_{n}, \hat{W}_{n} x_{n}\right)\right\|=\left|\hat{W}_{n} x_{n}\right|^{-2}\left\|W_{n} S\left(x_{n}, x_{n}\right)\right\| \\
& \leq\left\|W_{n}\right\|\left|\hat{W}_{n} x_{n}\right|^{-2}\left\|S\left(x_{n}, x_{n}\right)\right\| \leq\left\|W_{n}\right\|\left|\hat{W}_{n} x_{n}\right|^{-2} e .
\end{aligned}
$$

From $\left\|W_{n}\right\| \rightarrow\|W\|>0$ and $\left\|\hat{W}_{n} x_{n}\right\| \rightarrow \infty$ we get a contradiction to $d>0$. 
We therefore have proved that $\left(\hat{W}_{n} ; n \in N\right)$ is bounded and, consequently, $\omega$ contains a convergent subsequence. Hence, we may assume that $\omega$ converges to $(W, \hat{W})$. It remains to prove $\hat{W} \in \mathrm{Gl}_{c} U$. But $\hat{W} u=0$ implies $0=S(\hat{W} u, \hat{W} u)=W S(u, u)$; from $W \in$ Aut $K$ we conclude $0=S(u, u)$ and so $u=0$ by (1.12).

b) is an immediate consequence of a).

As a consequence we have

Theorem 1.2. For a Siegel domain $D(K, S)$ there are equivalent

(1) $D(K, S)$ is homogeneous,

(2) There exists a closed subgroup $\Gamma \subset$ Aut $K$ such that $(\Gamma, S)$ is admissible.

Proof. Use [36; Propositions 2.2 and 3.1] and Lemma 1.1.

5. For a Lie group $\Gamma$ we denote by Lie $\Gamma$ the Lie algebra of $\Gamma$.

To abbreviate notation we set for a Siegel domain $D(K, S)$

$$
\mathrm{g}(K, S)=\text { Lie Aut } D(K, S) \text {. }
$$

Where no confusion is possible we write $g$ for $g(K, S)$.

As usual, we identify $\mathrm{g}(K, S)$ with the Lie algebra of complete holomorphic vector fields on $D(K, S)$ [36; § 4].

Further, we identify all tangent spaces of $D(K, S)$ with $V^{c} \times U$; hence all elements of $g(K, S)$ are identified with holomorphic mappings from $D(K, S)$ into $V^{c} \times U$. For $X \in \mathfrak{g}(K, S)$ we therefore write

$$
X(z, u)=\left(X_{V}(z, u), X_{U}(z, u)\right) \in V^{c} \times U \quad \text { for }(z, u) \in D(K, S),
$$

where $X_{V}$ and $X_{U}$ are holomorphic mappings.

The Lie bracket of two vector fields $X$ and $Y$ of $g(K, S)$ can be computed as follows, $w:=(z, u) \in D(K, S)$,

$$
[X, Y](w)=\left.\frac{d}{d t}\{Y(w+t X(w))-X(w+t Y(w))\}\right|_{t=0} .
$$

As standard reference for results on $g(K, S)$ we use [29] and [36].

The following theorems are of special importance for this work and are therefore stated explicitly.

Here we use the adjoint representation $a d$ of $\mathrm{g}(K, S)$ and the vector field $\partial \in g(K, S), \partial(z, u)=\left(z, \frac{1}{2} u\right)$.

For a Siegel domain $D(K, S)$ and $\lambda \in R$ we denote by $g_{\lambda}(K, S)$ the eigenspace of ad $\partial$ in $\mathrm{g}(K, S)$. 
TheOREM 1.3 ([29]). a) $\mathrm{g}(K, S)$ consists of polynomials.

b) $\mathrm{g}(K, S)$ is the sum of the subspaces $\mathrm{g}_{\lambda}(K, S), \lambda=-1,-\frac{1}{2}, 0, \frac{1}{2}, 1$.

c) $\left[\mathfrak{g}_{\lambda}(K, S), \mathfrak{g}_{\mu}(K, S)\right] \subset \mathfrak{g}_{\lambda+\mu}(K, S)$.

The spaces $\mathfrak{g}_{\lambda}=\mathfrak{g}_{\lambda}(K, S)$ can be described in more detail. To do this we denote by $p_{k r}$ (resp. $q_{k r}$ ) polynomials in $(z, u)$ which are homogeneous of degree $k$ in $z$ and of degree $r$ in $u$.

Theorem 1.4 ([29]). a) $\mathfrak{g}_{-1}=\{(z, u) \mapsto(a, 0) ; a \in V\}$,

b) $\mathfrak{g}_{-\frac{1}{2}}=\{(z, u) \mapsto(2 i S(u, d), d) ; d \in U\}$,

c) $g_{0}=$ Lie Gl $D(K, S)$,

d) $\mathfrak{g}_{\frac{1}{2}} \subset\left\{(z, u) \mapsto\left(p_{11}(z, u), q_{10}(z, u)+q_{02}(z, u)\right)\right\}$,

e) $\mathfrak{g}_{1} \subset\left\{(z, u) \mapsto\left(p_{20}(z, u), q_{11}(z, u)\right)\right\}$.

Obviously, $\mathfrak{g}_{\frac{1}{2}}$ is contained in the vector space $\tilde{g}_{\frac{1}{2}}$ of all polynomials of type $\left(p_{11}, q_{10}+q_{\mathrm{N}_{2}}\right)$ and $\mathrm{g}_{1}$ is contained in the vector space $\tilde{\mathrm{g}}_{1}$ of all polynomials of type $\left(p_{20}, q_{11}\right)$. For a description of $g_{\frac{1}{2}}$ and $g_{1}$ it therefore suffices to find conditions which single out the elements of $g_{z}$ (resp. $g_{1}$ ) within $\tilde{g}_{\frac{1}{2}}$ (resp. $\tilde{g}_{1}$ ).

Theorem 1.5 ([37]). a) $\mathfrak{g}_{\frac{1}{2}}$ consists of all polynomials $X \in \tilde{\mathfrak{g}}_{\frac{1}{2}}$ which satisfy

$$
\left[X, \mathfrak{g}_{-\frac{1}{2}}\right] \subset \mathfrak{g}_{0}
$$

b) $g_{1}$ consists of all polynomials $X=\left(p_{20}, q_{11}\right) \in \tilde{g}_{1}$ which satisfy

$$
\begin{aligned}
& {\left[X, \mathfrak{g}_{-1}\right] \subset \mathfrak{g}_{0},} \\
& {\left[X, \mathfrak{g}_{-\frac{1}{2}}\right] \subset \mathfrak{g}_{\frac{1}{2}},} \\
& \operatorname{Im}\left(\operatorname{trace}\left(u \mapsto q_{11}(v, u)\right)\right)=0 \quad \text { for all } v \in V .
\end{aligned}
$$

Remark. For a tube domain we obviously have $\mathfrak{g}_{-\frac{1}{2}}=\mathfrak{g}_{\frac{1}{2}}=0$. The space $g_{1}$ has been described in [18] for this case (see also Theorem 2.8).

Finally, we consider the radical of $g(K, S)$.

TheOREM 1.6 ([29]). Let $\mathfrak{w}$ be the radical of $\mathrm{g}=\mathrm{g}(K, S)$.

a) $\mathfrak{w}=\mathfrak{w}_{-1}+\mathfrak{w}_{-\frac{1}{2}}+\mathfrak{w}_{0}, \mathfrak{w}_{\lambda}=\mathfrak{w} \cap \mathfrak{g}_{\lambda}$,

b) $\operatorname{dim}_{R} \mathfrak{g}_{\frac{1}{2}}=\operatorname{dim}_{R} \mathfrak{g}_{-\frac{1}{2}}-\operatorname{dim}_{R} \mathfrak{w}_{-\frac{1}{2}}$, $\operatorname{dim}_{R} \mathfrak{g}_{1}=\operatorname{dim}_{R} \mathfrak{g}_{-1}-\operatorname{dim}_{R} \mathfrak{w}_{-1}$.

In $\S 7$ the radical $\mathfrak{w}$ of $\mathfrak{g}$ will be described more precisely. 


\section{§2. A new description of homogeneous Siegel domains}

In this section, $D(K, S)$ denotes a homogeneous Siegel domain in $V^{C} \times U$.

1. It is known that for Siegel domains the Hilbert space of square integrable holomorphic functions has a reproducing kernel, the Bergmann kernel [1].

By [19; Proposition 5.1] we have

Theorem 2.1. Let $B\left(v_{1}, u_{1} ; v_{2}, u_{2}\right),\left(v_{k}, u_{k}\right) \in D(K, S), k=1,2$, denote the Bergmann kernel for $D(K, S)$, then there exists a map $\eta_{K S}: K \rightarrow R^{+}$such that

a) $\eta_{K S}$ can be extended holomorphically to $K+i V$,

b) $B\left(v_{1}, u_{1} ; v_{2}, u_{2}\right)=\eta_{K S}\left((2 i)^{-1}\left(v_{1}-\bar{v}_{2}\right)-S\left(u_{1}, u_{2}\right)\right)$.

Where no confusion is possible we write $\eta$ instead of $\eta_{K S}$.

In what follows we denote by $\Delta_{x}^{v}$ the directional derivation operator at $x$ in direction $v$.

A map $\chi: K \rightarrow R$ is called homogeneous (of degree $k$ ), if $\chi(\alpha x)=\alpha^{k} \chi(x)$ for all $\alpha>0$ and all $x \in K$.

The following result says that $\langle K, \eta, e\rangle$, for arbitrary $e \in K$, is a triple of $\mathscr{F}$ in the sense of $[14 ; \S 1,1]$.

LEMMA 2.2. a) $\eta$ is real analytic and homogeneous.

b) $\Delta_{x}^{v} \Delta_{x}^{v} \log \eta(x)>0$ for all $x \in K, v \in V, v \neq 0$.

c) $\eta(x)$ converges to $\infty$, when $x$ converges to a point of the (finite) boundary of $K$.

d) The group Aut $(K, \eta):=\{W \in$ Aut $K ; \eta(W x)=\alpha(W) \eta(x)$ for appropriate $\alpha(W)>0$ and all $x \in K\}$ acts transitively on $K$.

Proof. a) is obvious. To prove b), denote by $g_{(z, u)}$ the Bergmann metric at the point $(z, u) \in D(K, S)$. It is easy to show $g_{(i x, 0)}(v, v)=$ $\Delta_{x}^{v} \Delta_{x}^{v} \log \eta(x)$ for $x \in K, v \in V$. This implies b). Part c) follows from [30; Proposition 5.2] or [59; $\S 2$, Proposition 3]. Finally, choose $\Gamma \subset$ Aut $K$, such that $(\Gamma, S)$ is admissible. Thus, for $W \in \Gamma$ there exists a $\hat{W} \in \mathrm{Gl}_{C} U$ such that $(W, \hat{W}) \in \mathrm{Gl} D(K, S)$. By the properties of the Bergmann kernel we get $\eta(W x)=\operatorname{det} W^{-2}|\operatorname{det} \hat{W}|^{-2} \eta(x)$ for $x \in K$. Hence $\Gamma \subset \operatorname{Aut}(K, \eta)$ and (1.18) implies $d$ ).

Corollary 2.3. There exists a closed subgroup $\Gamma \subset$ Aut $K$ such that 
a) $(\Gamma, S)$ is admissible,

b) for all $W \in \Gamma$ there exists a $\hat{W} \in \mathrm{Gl}_{c} U$ such that $(W, \hat{W}) \in \mathrm{Gl} D(K, S)$ and $\eta(W x)=\operatorname{det} W^{-2}|\operatorname{det} \hat{W}|^{-2} \eta(x)$.

We remark that the argument of Lemma 2.2 shows that the first components of elements of Gl $D(K, S)$ are contained in $\operatorname{Aut}(K, \eta)$.

2. As remarked above, Lemma 2.2 means that $\langle K, \eta, e\rangle, e \in K$, is a triple of $\mathscr{F}$. In [14] and [17], there have been associated to such a triple several mathematical objects. As these objects will play an essential part in this paper we will assemble the most important definitions and some of the associated results.

We may suppose $\eta(e)=1$,

$$
\begin{aligned}
& \sigma(v, w):=\left.\Delta_{x}^{v} \Delta_{x}^{w} \log \eta(x)\right|_{x=e}, \quad v, w \in V . \\
& \sigma \text { is extended } C \text {-bilinear to } V^{c} . \\
& \sigma(h(x), v):=-\Delta_{x}^{v} \log \eta(x), \quad v \in V, \quad x \in K . \\
& \sigma(H(x) v, w):=\Delta_{x}^{v} \Delta_{x}^{w} \log \eta(x), \quad v, w \in V, \quad x \in K .
\end{aligned}
$$

Let $M$ be a finite dimensional vector space, $\tau$ a nondegenerate bilinear form on $M$ and $X$ an endomorphism of $M$. Then by $X^{\tau}$ we denote the adjoint endomorphism for $X$ with respect to $\tau$. Using this notation the following result follows from Lemma 2.2.

Lemma 2.4. a) $\sigma$ is a positive definite bilinear form on $V$.

b) $\quad h($ resp. $H)$ is real analytic and homogeneous of degree -1 (resp. $-2)$.

c) $H(e)=\mathrm{Id}, H(x)=H(x)^{\sigma}, H(x) v=-\Delta_{x}^{v} h(x)$ for $x \in K$.

d) $H(x)$ is positive definite (with respect to $\sigma$ ) for $x \in K$.

We use the $\sigma$-dual cone $K^{\sigma}$ (see (1.6)) and [17; Lemma 3.8, Theorems 4.2 and 4.4] and get

LEMMA 2.5. a) $h(K)=K^{\sigma}$,

b) $h: K \rightarrow K^{\sigma}$ is a birational diffeomorphism with the unique fixed point $e$.

Further, we recall (see [16; I, § 4]) the definition of the commutative algebra $\mathfrak{A}=\mathscr{A}(\eta, e)$ which is associated to $\langle K, \eta, e\rangle$. This algebra will be the main tool for the description of $\mathfrak{g}_{0}, \mathfrak{g}_{\frac{1}{2}}$ and $\mathfrak{g}_{1}$. 
We define the product in $\mathfrak{A}$ implicitly by

$$
\sigma(u v, w):=-\left.\frac{1}{2} \Delta_{x}^{u} \Delta_{x}^{v} \Delta_{x}^{w} \log \eta(x)\right|_{x=e}, \quad u, v, w \in V .
$$

The left multiplications in $\mathfrak{X}$ will be denoted by $A(v), v \in \mathfrak{A}$.

The complexification of $\mathfrak{Q}$ will be denoted by $\mathfrak{Q}^{C}$.

For the left multiplications of $\mathfrak{U}^{c}$ we write $A(z), z \in \mathfrak{U}^{c}$.

We have immediately

LEMMA 2.6. a) $\mathfrak{A}$ is a commutative algebra with unit e.

b) $\sigma(u v, w)=\sigma(u, v w)$ for $u, v, w \in V$.

c) $A(v)=A(v)^{\sigma}$ for $v \in V$.

Finally, we recall the definition of the mutation $\mathfrak{U}_{x}$ of $\mathfrak{A}$ by $x$. The product of $\mathfrak{A}_{x}$ is given by $(u, v) \mapsto(u x) v+u(x v)-x(u v)$. Obviously, $\mathfrak{A}_{x}$ is commutative but, in general, will have no unit element.

The importance of mutations for the description of $g$ arises from the following fact [18].

Theorem 2.7. For a tube domain $D(K)$ the elements of $\mathfrak{g}_{1}$ are all of type $z \mapsto A_{x}(z) z$ with appropriate $x \in V$.

Remark. For an arbitrary triple $F=\left\langle K_{F}, \eta_{F}, e_{F}\right\rangle$ of $\mathscr{F}$ we may define analogously a bilinear form $\sigma_{F}$, mappings $h_{F}$ and $H_{F}$ and an algebra $\mathfrak{U}_{F}$ (with unit $e_{F}$ ). Moreover, Lemma 2.5 and Lemma 2.6 still hold.

In what follows, however, we mainly deal with the triple $\langle K, \eta, e\rangle$ derived from a homogeneous Siegel domain. At the few places where we use different triples we will always make this clear by using the triple indicator as subscript.

3. We will now introduce some further notation which completes the alternative description of homogeneous Siegel domains.

By a simple argument we get

Lemma 2.8. The map $\rho: U \times U \rightarrow C$ which is defined by $\rho(u, w):=$ $\sigma(S(u, w), e)$ is a positive definite hermitian form on $U$.

We define a linear map $\varphi: V^{c} \rightarrow \operatorname{End}_{c} U$ by

$$
\rho(\varphi(x) u, w):=\sigma(S(u, w), x), \quad x \in V^{c}, \quad u, w \in U
$$

Further, we set

$$
\operatorname{Sym}(U, \rho):=\left\{X \in \operatorname{End}_{c} U ; X^{\rho}=X\right\},
$$


(2.8) $\operatorname{Pos}(U, \rho):=\{X \in \operatorname{Sym}(U, \rho) ; X$ positive definite with respect to $\rho\}$. By a simple computation we prove

LEMma 2.9. a) $\varphi(v)^{\rho}=\varphi(\bar{v})$ for $v \in V^{c}$,

b) $\varphi(x) \in \operatorname{Sym}(U, \rho)$ for $x \in V$,

c) $\varphi(x) \in \operatorname{Pos}(U, \rho)$ for $x \in K^{\sigma}$,

d) $\varphi(e)=\mathrm{Id}$.

e) For $W \in \mathrm{Gl}_{R} V$ and $\hat{W} \in \mathrm{Gl}_{C} U$ the following are equivalent

(i ) $W \in$ Aut $K$ and $\varphi\left(W^{\sigma} v\right)=\hat{W}^{\rho} \varphi(v) W$ for $v \in V^{c}$,

(ii) $(W, \hat{W}) \in \mathrm{Gl} D(K, S)$.

f) For $T \in \operatorname{End}_{R} V$ and $\hat{T} \in \operatorname{End}_{C} U$ the following are equivalent

(i ) $T \in$ Lie Aut $K$ and $\varphi\left(T^{o} v\right)=\hat{T}^{\rho} \varphi(v)+\varphi(v) \hat{T}$ for $v \in V^{c}$,

(ii) $(T, \hat{T}) \in$ Lie Gl $D(K, S)$.

4. We want to apply the results of [15] on J-morphisms of homogeneous cones (for definitions we refer to [15]). We therefore define a triple $M_{K S}:=(F, \varphi, \hat{F})$ where

$$
F:=\left\langle K^{\sigma}, \iota\left(K^{o} ;\right), e\right\rangle,
$$

$$
\left.\hat{F}:=\langle\operatorname{Pos}(U, \rho), \iota(\operatorname{Pos} U, \rho) ; \quad)^{a}, \mathrm{Id}\right\rangle, \quad a:=\frac{1}{2}\left[\operatorname{dim}_{C} U\right]^{-1} .
$$

As in $[15 ; 4]$ the "invariants" are normalized as to take the value 1 at $e$ (resp. Id).

It is easily seen $c(\operatorname{Pos}(U, \rho) ; X)^{a}=|\operatorname{det} X|^{-\frac{1}{2}}$.

Where no confusion is possible we write $M$ instead of $M_{K S}$.

From Lemma 2.9 we easily derive

LEMMA 2.10. $M$ is a J-morphism of homogeneous cones.

By $[15 ;(1.6)]$ we form the new triple of $\mathscr{F}$

$$
G\left(M_{K S}\right):=\left\langle K^{\sigma}, \gamma_{K S}, e\right\rangle
$$

where

$$
\gamma_{K S}(y):=\iota\left(K^{\sigma} ; y\right)[\operatorname{det} \varphi(y)]^{-\frac{1}{2}}, \quad y \in K^{\sigma} .
$$

We write $\gamma$ instead of $\gamma_{K S}$ where no confusion is possible.

We recall that in $[17 ; \S 8]$ to each triple $Q$ of $\mathscr{F}$ there was defined a triple $Q_{\sigma}$. To simplify notation we use $\tilde{Q}$ instead of $Q_{\sigma}, \tilde{\gamma}$ instead of $\gamma_{\sigma}$ etc.

LEMMA 2.11. a) $[\tilde{\gamma}(x)]^{2}=\eta(x), x \in K$, 
b) $\eta(x)=[\iota(K ; x)]^{2} \operatorname{det} \varphi(h(x)), x \in K$,

c) $\eta$ is a rational map.

Proof. Set $\psi(x):=[\eta(x)]^{-1}[\iota(K ; x)]^{2} \operatorname{det} \varphi(h(x))$ where $\iota(K ; e)=1$ and choose a subgroup $\Gamma \subset$ Aut $K$ such that $(\Gamma, S)$ is admissible. Then it is easy to verify $\psi(W x)=\psi(x)$ for $W \in \Gamma$. This proves b). For the triple $N:=\langle K, \eta, e\rangle$ we compute $\tilde{N}$ and get $\tilde{N}=\left\langle K^{o}, \gamma^{2}, e\right\rangle$ because of $\tilde{\eta}(y)=$ $[\gamma(y)]^{2}$. Hence, for $G=G\left(M_{K S}\right)$ we get $\sigma_{N}=\sigma_{\tilde{N}}=2 \sigma_{G}$ and $h_{N}^{-1}=h_{\tilde{N}}=h_{G}$. Part a) follows. By Lemma 2.5 and [17; Lemma 3.8] we derive that $h$ and $\iota(K ; \quad)^{2}$ are rational.

Corollary 2.12. Set $N:=\langle K, \eta, e\rangle$ and $G:=G\left(M_{K S}\right)$ then

a) $\mathfrak{U}_{N}=\mathfrak{U}_{G}, \sigma=\sigma_{N}=2 \sigma_{G}, h_{N}=h_{\tilde{G}}, H_{N}=H_{\tilde{G}}$.

b) $\operatorname{Aut}(K, \eta)=\operatorname{Aut}\left(\left[K_{G}\right]^{\sigma a}, \tilde{\eta}_{G}\right)$.

Proof. We frequently use the results of $[17 ; \S 8]$. First we have $\sigma_{\tilde{G}}=$ $\sigma_{G}=\frac{1}{2} \sigma_{\tilde{N}}=\frac{1}{2} \sigma_{N}=\frac{1}{2} \sigma$. This implies $h_{N}=h_{\tilde{G}}$ by part a) of Lemma 2.11. Hence $H_{N}=H_{\tilde{G}}$ and $\mathfrak{U}_{N}=\mathfrak{U}_{\tilde{G}}=\mathfrak{U}_{G}$ and a) is proved. Further, we get $\left[K_{G}\right]^{\sigma a}=\left(K^{\sigma}\right)^{\sigma}=K$. We apply again Lemma 2.11 to see $\tilde{\gamma}=\tilde{\eta}_{G}=\eta^{\frac{1}{2}}$. Part b) follows.

Remark. By Lemma 2.10 we get a $J$-morphism $M$ with associated triple $G=\left\langle K^{\sigma}, \gamma, e\right\rangle$. We apply the results of [15] and so derive a description of Lie Aut $\left(\left[K_{G}\right]^{\sigma}{ }^{\sigma}, \tilde{\eta}_{G}\right)=\operatorname{Lie} \operatorname{Aut}(K, \eta)$. This will be carried out in detail in $\S 3$.

5. In this paragraph we explain how one can compute $\eta=\eta_{K S}$ explicitly. We choose an arbitrary positive definite bilinear form $\tau$ on $V$ and extend $\tau$ to a $C$-bilinear form on $V^{c}$. We choose $x_{0} \in K^{\tau}$ and put $\kappa(u, w):=\tau\left(S(u, w), x_{0}\right)$ for $u, w \in U$. As in Lemma 2.8 we see that $\kappa$ is a positive definite hermitian form on $U$.

We define a map $\psi: V^{c} \rightarrow \operatorname{End}_{c} U$ as in (2.6) by $\kappa(\psi(x) u, w)=$ $\tau(S(u, w), x), x \in V^{c}, u, w \in U$. As in Lemma 2.9 we get $\psi\left(W^{\tau} x\right)=\hat{W}^{x} \psi(x) \hat{W}$ for each $(W, \hat{W}) \in \mathrm{Gl} D(K, S)$. By [17; Theorem 4.2] we know that $h_{z}$, given by $\tau\left(h_{\tau}(x), v\right)=-\Delta_{x}^{v} \log \iota(K ; x)$, is a diffeomorphism from $K$ onto $K^{\tau}$ and for all $W \in$ Aut $K$ and all $x \in K$ the equality $h_{\tau}(W x)=\left[W^{\tau}\right]^{-1} h_{\tau}(x)$ holds. We put $\chi(x):=[\iota(K ; x)]^{2} \operatorname{det} \psi\left(h_{\tau}(x)\right)$ and get $\chi(W x)=|\operatorname{det} W \operatorname{det} \hat{W}|^{-2} \chi(x)$ for $x \in K,(W, \hat{W}) \in \mathrm{Gl} D(K, S)$. As in Lemma 2.11, we have that $\chi$ and $\eta_{K S}$ coincide (up to a positive constant factor).

6. In this paragraph we present different descriptions for the class 
of homogeneous Siegel domains.

By $\operatorname{Si}(V, U)$ we denote the set of triples $(K, S, e)$ associated to homogeneous Siegel domains $D(K, S)$ in $V^{c} \times U, e \in K$.

We write $M o(V, U)$ for the set of tuples $(K, \chi, e, \varphi, \rho)$ which satisfy the following six conditions.

(2.13) $K$ is a regular cone in $V$ and $e \in K$.

(2.14) $\rho$ is a positive definite hermitian form on $U$.

(2.15) $\varphi: V \rightarrow \operatorname{Sym}(U, \rho)$ is a linear map satisfying $\varphi(e)=\mathrm{Id}$.

(2.16) The triple $N=(K, \chi, e)$ is in $\mathscr{F}$ and $\varphi(x)$ is positive definite (with respect to $\rho$ ) on $K^{\tau}, \tau=\sigma_{N}$.

(2.17) There exists a closed subgroup $\Gamma \subset$ Aut $K$ which operates transitively on $K$ and which has the property that to each $W \in \Gamma$ there exists a $\hat{W} \in \mathrm{Gl}_{c} U$ such that the identity $\varphi\left(W^{o} x\right)=$ $\hat{W}^{\rho} \varphi(x) \hat{W}$ holds for all $x \in V$.

(2.18) Up to a positive constant factor $\chi(x)$ coincides with $[\iota(K ; x)]^{2} \operatorname{det} \varphi\left(h_{N}(x)\right)$ and satisfies $\chi(e)=1$.

Finally, by $\mathfrak{M}(V, U)$ we denote the set of pairs $(M, \rho)$ where $\rho$ is a positive definite hermitian form on $U$ and, using the notation of [15; (8.4)], $M$ is an element of $\mathfrak{M}(V, \operatorname{Sym}(U, \rho), \lambda), \lambda(A)=\frac{1}{2}$ trace $A$. (Here the product. of the Jordan algebra $\operatorname{Sym}(U, \rho)$ is given by $(A, B) \mapsto \frac{1}{2}(A B+B A)$.)

In the following theorem we prove that the sets $\operatorname{Si}(V, U), M o(V, U)$ and $\mathfrak{M}(V, U)$ are essentially the same. Hence, via [15; Theorem 8.5] we get an algebraic description of all homogeneous Siegel domains. This algebraic classification of homogeneous Siegel domains contains implicitly a construction procedure for each homogeneous Siegel domain from lower dimensional ones. Moreover, the construction from lower dimensional domains of a domain $D(K, S)$ is uniquely determined by $D(K, S)$.

TheOREM 2.13. There exist canonical bijections between $\operatorname{Si}(V, U), \quad \operatorname{Mo}(V, U), \quad \mathfrak{M}(V, U)$.

Proof. a) We define $f_{1}: S i(V, U) \rightarrow M o(V, U)$ to be the map which associates to a triple $(K, S, e)$ of $\operatorname{Si}(V, U)$ the tuple $\left(K, \eta_{K S}, e, \varphi, \rho\right)$, where $\eta_{K S}, \varphi$ and $\rho$ are defined by Theorem 2.1, Lemma 2.8 and (2.6). By the results of this paragraph we see that $f_{1}$ is well defined. 
b) By $g_{1}: M o(V, U) \rightarrow \operatorname{Si}(V, U)$ we denote the map which associates to a tuple $(K, \chi, e, \varphi, \rho)$ of $M o(V, U)$ the triple $(K, S, e)$, where $S$ is defined by the equation $\sigma_{N}(S(u, w), x)=\rho(\varphi(x) u, w), N=\langle K, \chi, e\rangle$. It is easy to check that $S$ is a $K$-hermitian form and $D(K, S)$ is a homogeneous Siegel domain. This shows that $g_{1}$ is well defined. Further, by definition of $\sigma$ and equation (2.6) we have $g_{1} \circ f_{1}=\mathrm{Id}$.

c) To prove $f_{1} \circ g_{1}=\operatorname{Id}$ we start with $(K, \chi, e, \varphi, \rho) \in M o(V, U)$ and have $S$ defined by $\sigma_{N}(S(u, w), x)=\rho(\varphi(x) u, w)$. We use (2.17) and (2.18) to verify $\chi=\eta_{K S}$. This implies $\sigma=\sigma_{N}$ and the hermitian form $\rho$ defined by Lemma 2.8 for $(K, S, e)$ coincides with the given one. By (2.6) it follows that $\varphi$ is the function defined by means of $(K, S, e)$. Hence $f_{1} \circ g_{1}=I d$.

d) We define a map $f_{2}: M o(V, U) \rightarrow \mathfrak{M}(V, U)$ by attaching to a tuple $(K, \chi, e, \varphi, \rho) \in M o(V, U)$ the pair $(M, \rho), M=(F, \varphi, \hat{F})$ with $F$ (resp. $\hat{F}$ ) defined by (2.9) (resp. (2.10)) where $\sigma=\sigma_{N}$. Obviously, $M$ coincides with $M_{K S}$ for the homogeneous Siegel domain $g_{1}(K, \chi, e, \varphi, \rho)$. Hence, by Lemma 2.10 we know that $M$ is a $J$-morphism. The choice of the exponent $a$ in (2.10) gives $(M, \rho) \in \mathfrak{M}(V, U)$.

e) Finally, we define a map $g_{2}: \mathfrak{M}(V, U) \rightarrow M o(V, U)$. Let $(M, \rho) \in$ $\mathfrak{M}(V, U), M=(F, \varphi, \hat{F})$. From the definition of $\mathfrak{M}(V, U)$ we know $M \in$ $\mathfrak{M}(V, \operatorname{Sym}(U, \rho), \lambda), \lambda(A)=\frac{1}{2} \operatorname{trace} A, A \in \operatorname{Sym}(U, \rho)$. We put $R:=G(M)$, $\sigma=\sigma_{R}, K:=\left(K_{F}\right)^{\sigma}, \chi:=\tilde{\gamma}_{M}, e:=e_{F}$ and $g_{2}(M, \rho):=(K, \chi, e, \varphi, \rho)$. It is clear that (2.13), (2.14) and (2.15) are satisfied. By [15; Lemma 1.1] we know that $R$ is a triple of $\mathscr{F}$. Hence $\tilde{R}=\langle K, \chi, e\rangle$ is a triple of $\mathscr{F}$ and we have $\sigma_{\tilde{R}}=\sigma_{R}=\sigma$ by $[17 ; \S 8]$. From the definition of $M$ we get that $\varphi(x)$ is positive definite for $x \in K_{F}=K^{\sigma}$. This proves (2.16). Condition (2.17) is easily derived from the definition of a $J$-morphism. To verify (2.18) we first note that by the definition of $\mathfrak{M}(V, U)$ we have $F=\left\langle K_{F}, \iota\left(K_{F} ;\right), e_{F}\right\rangle$ and $\hat{F}=\left\langle\operatorname{Pos}(U, \rho), \eta^{2}, \mathrm{Id}\right\rangle$ where $\eta^{\lambda}(X)=\iota(\operatorname{Pos}(U, \rho) ; X)^{r}, r=\frac{1}{2}\left[\operatorname{dim}_{C} U\right]^{-1}$. This implies $\eta^{\lambda}(\varphi(x))=[\operatorname{det} \varphi(x)]^{-\frac{1}{2}}$ and by the definition of $\gamma_{M}[15 ;(1.5)]$ we get $\gamma_{M}(x)=\iota\left(K_{F} ; x\right)[\operatorname{det} \varphi(x)]^{-\frac{1}{2}}$. From [17; Theorem 4.2] we know that $h_{R}$ is a diffeomorphism from $K_{F}$ onto $K$. Further, by [17; §8] we have $h_{\tilde{R}}=\left[h_{R}\right]^{-1}$. Hence, $\chi(x)=\tilde{\gamma}_{M}\left(x=\gamma_{M}\left(h_{\tilde{R}}(x)\right)^{-1}=\left[\iota\left(K_{F} ; h_{\tilde{R}}(x)\right)\right]^{-1}\left[\operatorname{det} \varphi\left(h_{\tilde{R}}(x)\right)\right]^{\frac{1}{2}}\right.$. This proves (2.18).

f) It remains to prove $f_{2} \circ g_{2}=\mathrm{Id}$ and $g_{2} \circ f_{2}=\mathrm{Id}$. We show that $f_{2}$ is injective and that $f_{2} \circ g_{2}=\mathrm{Id}$ holds. Suppose we have $f_{2}(K, \chi, e, \varphi, \rho)=$ $f_{2}\left(K^{\prime}, \chi^{\prime}, e^{\prime}, \varphi^{\prime}, \rho^{\prime}\right)$. Then, by definition of $f_{2}$, we get $(F, \varphi, \hat{F})=\left(F^{\prime}, \varphi^{\prime}, \hat{F}^{\prime}\right)$ and $\rho=\rho^{\prime}$. This implies $\varphi=\varphi^{\prime}, e=e^{\prime}$ and $K^{\sigma}=K^{\prime \sigma^{\prime}}$ where $\sigma=\sigma_{N}, N=$ 
$\langle K, \chi, e\rangle$ and $\sigma^{\prime}=\sigma_{N^{\prime}}, N^{\prime}=\left\langle K^{\prime}, \chi^{\prime}, e\right\rangle$. We use (2.18) and $K^{\sigma}=K^{\prime \sigma^{\prime}}$ to see $\tilde{\chi}=\tilde{\chi}^{\prime}$. This implies $\sigma=\sigma^{\prime}$, hence $K=K^{\prime}$ and $h_{N}^{-1}=h_{N^{\prime}}^{-1}$. From this we get $\chi=\chi^{\prime}$ and $f_{2}$ is injective. Let now $(M, \rho) \in \mathfrak{M}(V, U)$. It is easy to verify that it suffices to prove $K_{F}=\left(K_{F}^{o}\right)^{\tau}$ with $\sigma=\sigma_{G(M)}$ and $\tau=\sigma_{N}$, $N=\left\langle K_{F}^{\sigma}, \tilde{\gamma}_{M}, e_{F}\right\rangle$. Because of $N=\widetilde{G(M)}$ we have $\sigma=\tau$. Hence the assertion and the theorem is proved.

\section{§3. Description of $g_{0}(K, S)$}

In this section we give a detailed description of $\mathfrak{g}_{0}=$ Lie Gl $D(K, S)$ for homogeneous Siegel domains. We use the notation introduced in the preceding sections. We mainly draw from [15].

By $D(K, S)$ we always mean a homogeneous Siegel domain in $V^{C} \times U$.

1. As remarked before, because of Lemma 2.11 we may apply the results of [15]. By Corollary 2.13 we thus get a description of $\mathfrak{m}:=$ Lie $\operatorname{Aut}\left(K, \eta_{K S}\right)$.

Let $\mathfrak{U}$ denote the algebra which has been defined from $\left\langle K, \eta_{K S}, e\right\rangle$ in $\S 2.2$. Corollary 2.13 shows that we have $\mathfrak{U}=\mathfrak{U}_{G\left(M_{K S}\right)}=\mathfrak{A}_{\overparen{G\left(M_{K S}\right)}}$.

For idempotents $e_{11}, \cdots, e_{q q}$ of $\mathfrak{A}$ we set

$$
\begin{aligned}
& \mathfrak{A}_{i i}:=\left\{x \in \mathfrak{X} ; e_{i i} x=x\right\} . \\
& \mathfrak{A}_{i j}:=\mathfrak{U}_{j i}:=\left\{x \in \mathfrak{A} ; e_{i i} x=\frac{1}{2} x, e_{j i} x=\frac{1}{2} x\right\}, \quad 1 \leq i<j \leq q . \\
& \mathfrak{U}^{(k)}:=\bigoplus_{k \leq i \leq j \leq q} \mathfrak{U}_{i j} . \\
& A^{(k)}(x):=\left.A(x)\right|_{\mathscr{Y}(k)} .
\end{aligned}
$$

By Lemma 2.11 we may apply the map $\mathscr{H}_{1}$ of $[15 ;(8.6)]$ to $M_{K S}$. Hence, from [15; Theorem 8.5] we know that $\mathscr{H}_{1}\left(M_{K S}\right)$ is the algebraic equivalent of $M_{K S}$.

By the definition of $\mathscr{H}_{1}\left(M_{K S}\right)$ we get

Theorem 3.1. There exist uniquely determined $q \in N$ and idempotents $e_{11}, \cdots, e_{q q}$ of $\mathfrak{A}$ such that
a) $\mathfrak{U}=\oplus_{1 \leq i \leq j \leq q} \mathfrak{X}_{i j}$,
b) $\mathfrak{A}_{i j} \mathfrak{A}_{k s}=0$ if $\{i, j\} \cap\{k, s\}=\emptyset$, $\mathfrak{A}_{i j} \mathfrak{U}_{j k} \subset \mathfrak{A}_{j k}$ if $i \neq k$, $\mathfrak{A}_{i j} \mathfrak{U}_{i j} \subset \mathfrak{U}_{i i}+\mathfrak{U}_{j j}$.
c) $\mathfrak{U}_{e_{k k}}$ is a Jordan algebra,
d) $A_{e_{s s}(k)}^{(k)}\left(x_{s n}\right)$ is a derivation of $\mathfrak{Q}_{e_{k k}^{(k)}}^{(k)}$ if $1 \leq k<s \leq n \leq q$ and $x_{s n} \in \mathfrak{U}_{s n}$. 
In what follows, where we use $e_{k k}$ or $\mathfrak{U}_{i j}$ we tacitly refer to Theorem 3.1.

We are going to describe $\mathfrak{m}$. We put

$$
\begin{aligned}
& \mathfrak{m}_{i j}:=\left\{A_{e_{i i}}\left(x_{i j}\right) ; x_{i j} \in \mathfrak{A}_{i j}\right\} \quad \text { if } 1 \leq i<j \leq q . \\
& \mathfrak{m}_{i i}:=\text { the Lie algebra generated by }\left\{A\left(x_{i i}\right) ; x_{i i} \in \mathfrak{U}_{i i}\right\} . \\
& \mathfrak{a}:=\left\{D ; D \text { derivation of } \mathfrak{A}, D \mathfrak{U}_{i i}=0 \text { for all } i\right\} .
\end{aligned}
$$

As in $[15 ; 7]$ we split the reductive Lie algebra $\mathfrak{a}$ (resp. $\mathfrak{m}_{i i}$ into the direct sum of its center and a semisimple summand

$$
\begin{aligned}
& \mathfrak{m}_{i i}=\mathfrak{z}_{i i}+\mathfrak{h}_{i i}, \\
& \mathfrak{a}=\mathfrak{a}_{\mathfrak{z}}+\mathfrak{a}_{\mathfrak{g}} .
\end{aligned}
$$

Further, we set

$$
\mathfrak{n}:=\bigoplus_{1 \leq i<j \leq q} \mathfrak{m}_{i j} .
$$

From [15; Theorem 7.3] we conclude

Theorem 3.2. a) Lie Aut $\left(K, \eta_{K S}\right)=\mathfrak{n}+\mathfrak{a}+\oplus_{i=1}^{q} \mathfrak{m}_{i l}$ (direct sum of vector spaces).

b) $\mathfrak{a}_{\mathfrak{z}} \oplus \oplus_{i=1}^{q} \hat{o}_{i i}$ is an abelian algebraic Lie algebra consisting of semisimple endomorphisms (direct sum of Lie algebras).

c) $\mathfrak{a}_{\mathfrak{h}} \oplus \oplus_{i=1}^{q} \mathfrak{h}_{i i}$ is a maximal semisimple subalgebra of LieAut $\left(K, \eta_{K S}\right)$ (direct sum of Lie algebras).

d) $\mathfrak{n}+\mathfrak{a}_{\mathfrak{z}}+\oplus_{i=1}^{q} \mathfrak{z}_{i i}$ is the radical of Lie Aut $\left(K, \eta_{K S}\right)$ (direct sum of vector spaces).

e) $\mathfrak{n}$ is the maximal ideal of Lie Aut $\left(K, \eta_{K S}\right)$ consisting of nilpotent endomorphisms.

Next we characterize the spaces $\mathfrak{A}_{i i}$ and $\mathfrak{X}:=\bigoplus_{i=1}^{q} \mathfrak{A}_{i i}$. From [15; Lemma 6.1, Theorems 6.3 and 7.3] we get

TheOREM 3.3. a) $\mathfrak{X}=\bigoplus_{i=1}^{q} \mathfrak{A}_{i i}=\left\{x \in \mathfrak{A} ; A(x) \in \operatorname{Lie} \operatorname{Aut}\left(K, \eta_{K S}\right)\right\}$,

b) $\mathfrak{U}_{k k}=\left\{a \in \mathfrak{U}^{(k)} ; A_{a}(v) \in \operatorname{Lie} \operatorname{Aut}\left(K, \eta_{K S}\right)\right.$ for all $\left.v \in \mathfrak{U}^{(k)}\right\}$.

2. Sometimes it is convenient to use a coarser splitting of $\mathfrak{U}$. We set

$$
\begin{aligned}
& \mathfrak{U}_{1}:=\mathfrak{U}_{11}, \\
& \mathfrak{U}_{\frac{1}{2}}:=\bigoplus_{1<k \leq q}^{\bigoplus} \mathfrak{U}_{1 k},
\end{aligned}
$$




$$
\mathfrak{A}_{0}:=\bigoplus_{2 \leq i \leq j \leq q} \mathfrak{A}_{i j} \cdot
$$

Further, to simplify notation we put

$$
\begin{aligned}
& c:=c_{1}:=e_{11} \text { and } \quad c_{0}:=e-c_{1} . \\
& A_{\frac{1}{2}}\left(x_{i}\right):=\left.A\left(x_{i}\right)\right|_{\mathfrak{q}_{\frac{1}{2}}}, \quad x_{i} \in \mathfrak{A}_{i}, \quad i=0,1 .
\end{aligned}
$$

We use [14; Corollary 7.6] and get

TheOREM 3.4. a) $A_{\frac{1}{2}}: \mathfrak{U}_{0} \rightarrow \operatorname{End}_{R} \mathfrak{Y}_{\frac{1}{2}}$ is injective,

b) $\mathfrak{U}_{0}$ is generated (as a vector space) by $\left\{c_{0}\left(x_{\frac{1}{2}}^{2}\right) ; x_{\frac{1}{2}} \in \mathfrak{U}_{\frac{1}{2}}\right\}$.

3. Up to now we have drawn from properties of $\mathscr{H}_{1}\left(M_{K S}\right)$ which are related to $V$. For the description of $\mathrm{g}_{0}=$ Lie $\mathrm{Gl} D(K, S)$ we need precise information on how $\varphi$ "behaves" with respect to the spaces $\mathfrak{A}_{i j}$.

We first note an easy consequence of (2.10).

LEMMA 3.5. Let $M_{K S}=(F, \varphi, \hat{F})$ and $\hat{\mathfrak{U}}=\mathfrak{A}_{\hat{F}}$.

a) The product in $\hat{\mathfrak{A}}=\operatorname{Sym}(U, \rho)$ is given by $(\hat{X}, \hat{Y}) \mapsto \frac{1}{2}(\hat{X} \hat{Y}+\hat{Y} \hat{X})$,

b) $\quad \hat{\sigma}(\hat{X}, \hat{Y}):=\sigma_{\hat{F}}(\hat{X}, \hat{Y})=\frac{1}{2} \operatorname{trace} \hat{X} \hat{Y}$.

In this paper we always provide $\operatorname{Sym}(U, \rho)$ with the product of Lemma 3.5.

We use Lemma 3.5 and derive from [15; Theorem 5.5]

Lemma 3.6. For all $x \in \mathfrak{X}$ and all $v \in \mathfrak{A}$ we have

$$
\varphi(x v)=\frac{1}{2}(\varphi(x) \varphi(v)+\varphi(v) \varphi(x)) .
$$

Let $d_{1}, \cdots, d_{n}$ be a complete system of orthogonal idempotents of $\mathfrak{U}$ which are contained in $\mathfrak{X}$ (we abbreviate this by CSI). Consider the Peirce decomposition $\mathfrak{U}=\oplus_{1 \leq i \leq j \leq n} \widetilde{\mathfrak{A}}_{i j}$ of $\mathfrak{A}$ with respect to the CSI $d_{1}, \cdots, d_{n}$. (For definition and properties of a Peirce decomposition we refer to [18].)

Lemma 3.7. Let $d_{1}, \cdots, d_{n}$ be a CSI and $\mathfrak{A}=\bigoplus_{1 \leq i \leq j \leq n} \widetilde{\mathfrak{A}}_{i j}$ the Peirce decomposition of $\mathfrak{X}$ with respect to $d_{1}, \cdots, d_{n}$. Then for $1 \leq i, j \leq n$ and $x_{j j} \in \hat{\mathfrak{A}}_{j j}$ we have

$$
\varphi\left(d_{i}\right) \varphi\left(x_{j j}\right)=\varphi\left(x_{j j}\right) \varphi\left(d_{i}\right)=\delta_{i j} \varphi\left(x_{j j}\right) .
$$

Proof. From Lemma 3.6 we get $2 \delta_{i j} \varphi\left(x_{j j}\right)=\varphi\left(d_{i}\right) \varphi\left(x_{j j}\right)+\varphi\left(x_{j j}\right) \varphi\left(d_{i}\right)$. In particular $\varphi\left(d_{i}\right)=\varphi\left(d_{i}\right)^{2}$ holds. By $\hat{X} \circ \hat{Y}$ we denote the product in $\operatorname{Sym}(U, \rho)$ and verify $\varphi\left(d_{i}\right) \varphi\left(x_{j j}\right) \varphi\left(d_{i}\right)=2 \varphi\left(d_{i}\right) \circ\left(\varphi\left(d_{i}\right) \circ \varphi\left(x_{j j}\right)\right)-\varphi\left(d_{i}\right) \circ \varphi\left(x_{j j}\right)$ $=\delta_{i j} \varphi\left(x_{j j}\right)$. The equality $\delta_{i j} \varphi\left(x_{j j}\right)=2 \varphi\left(d_{i}\right) \frac{1}{2}\left\{\varphi\left(x_{j j}\right) \varphi\left(d_{i}\right)+\varphi\left(d_{i}\right) \varphi\left(x_{j j}\right)\right\}-$ $\varphi\left(d_{i}\right) \varphi\left(d_{i}\right) \varphi\left(x_{j j}\right)=\left(2 \delta_{i j}-1\right) \varphi\left(d_{i}\right) \varphi\left(x_{j j}\right)$. Hence $\varphi\left(d_{i}\right) \varphi\left(x_{j j}\right)=\delta_{i j} \varphi\left(x_{j j}\right)$. The 
assertion follows as $\varphi\left(x_{j j}\right)$ is selfadjoint with respect to $\rho$ by Lemma 2.10 .

CoRollary 3.8. With the notation of Lemma 3.7 and $1 \leq i, j \leq n$ we get

a) $\varphi\left(d_{i}\right)$ is an orthogonal projection (with respect to $\rho$ ),

b) $\varphi\left(d_{i}\right) \varphi\left(d_{j}\right)=\delta_{i j} \varphi\left(d_{i}\right), \sum_{k=1}^{n} \varphi\left(d_{k}\right)=\mathrm{Id}$.

c) $\varphi\left(x_{i i}\right) \varphi\left(x_{j j}\right)=0$ for $x_{i i} \in \widetilde{\mathfrak{A}}_{i i}, x_{j j} \in \widetilde{\mathfrak{A}}_{j j}$ if $i \neq j$.

Proof. We only have to prove c). But $\varphi\left(x_{i i}\right) \varphi\left(x_{j j}\right)=\varphi\left(x_{i i}\right) \varphi\left(d_{i}\right) \varphi\left(x_{j j}\right)$ $=0$ by Lemma 3.7 .

Lemma 3.9. Let $d_{1}, \cdots, d_{n}$ and $\widetilde{\mathfrak{A}}_{i j}$ as in Lemma 3.7. We set $U_{i}:=$ $\varphi\left(d_{i}\right) U$. Then we have

a) $U=\oplus_{i=1}^{q} U_{i}$,

b) $\varphi\left(x_{i j}\right) U_{k} \subset \delta_{i k} U_{j}+\delta_{j k} U_{i}$ for $1 \leq i, j, k \leq n, x_{i j} \in \widetilde{\mathfrak{A}}_{i j}$.

c) $S\left(U_{i}, U_{j}\right) \subset \widetilde{\mathfrak{A}}_{i j}$ for $1 \leq i, j \leq n$.

Proof. a) is clear by Corollary 3.8. To prove b) we first note that the case $i=j$ follows from Lemma 3.7. In the case $i \neq j$ we may assume $i \neq k$. Hence $\varphi\left(x_{i j}\right) U_{k}=2 \varphi\left(d_{i} x_{i j}\right) U_{k}=\varphi\left(d_{i}\right) \varphi\left(x_{i j}\right) U_{k}+\varphi\left(x_{i j}\right) \varphi\left(d_{i}\right) U_{k}=$ $\varphi\left(d_{i}\right) \varphi\left(x_{i j}\right) U_{k}$ and $\varphi\left(x_{i j}\right) U_{k} \subset U_{i}$. If, additionally, $j \neq k$ then $\varphi\left(x_{i j}\right) U_{k} \subset U_{i}$ $\cap U_{j}=0$. b) follows. Finally, from Lemma 2.10 and Lemma 3.6 we get $\left(A\left(d_{i}\right), \frac{1}{2} \varphi\left(d_{i}\right)\right) \in$ Lie Gl $D(K, S)$. This implies $d_{i} S\left(u_{i}, u_{j}\right)=\frac{1}{2} S\left(\varphi\left(d_{i}\right) u_{i}, u_{j}\right)+$ $\frac{1}{2} S\left(u_{i}, \varphi\left(d_{i}\right) u_{j}\right)$. We put $x:=S\left(u_{i}, u_{j}\right)$ and get $d_{i} x=\frac{1}{2} x, d_{j} x=\frac{1}{2} x$ if $i \neq j$ and $d_{i} x=x$ if $i=j$. This proves $c$ ).

4. In this paragraph we exploit another property of $\varphi$ and derive a description of $\mathrm{g}_{0}$.

From [15; Lemma 8.1] we know that

$$
\tilde{\varphi}\left(A_{e_{s s}}\left(x_{s j}\right)\right):=\hat{A}_{\varphi\left(e_{s s}\right)}\left(\varphi\left(x_{s j}\right)\right), \quad 1 \leq j \leq s \leq q, \quad x_{s j} \in \mathfrak{A}_{s j}
$$

defines a homomorphism from the Lie algebra $\mathfrak{m}_{0}^{*}:=\left\{T^{\sigma} ; T \in \mathfrak{m}_{0}\right\}, \mathfrak{m}_{0}:=$ $\oplus_{1 \leq i \leq j \leq q} \mathfrak{m}_{i j}$, into the Lie algebra Lie Str $\hat{\mathfrak{A}}$, the structure group of $\hat{\mathfrak{A}}$ (for definition see [5; IX, §5]).

Let $1 \leq j \leq s \leq q$ and $x_{j s} \in \mathfrak{A}_{j s}$; then we use Lemma 3.3 and (3.16) and get by a straightforward computation

$$
\varphi\left(\left[A_{e_{j j}}\left(x_{j s}\right)\right]^{\sigma} v\right)=\left[\frac{1}{2} \varphi\left(e_{s s}\right) \varphi\left(x_{j s}\right)\right]^{\rho} \varphi(v)+\varphi(v)\left[\frac{1}{2} \varphi\left(e_{s s}\right) \varphi\left(x_{j s}\right)\right] .
$$

On the other hand we know that the map

$$
\xi:\left(\operatorname{End}_{C} U\right)^{-} \longrightarrow \operatorname{Lie} \operatorname{Str} \hat{\mathcal{A}}, \quad \xi(\hat{T})(\hat{X}):=-\hat{T}^{\circ} \hat{X}-\hat{X} \hat{T}
$$


is an isomorphism of Lie algebras (where $\left(\operatorname{End}_{c} U\right)^{-}$denotes the usual Lie algebra on $\operatorname{End}_{C} U$ ).

We define a map $\psi: \mathfrak{m}_{0} \rightarrow\left(\operatorname{End}_{c} U\right)^{-}$by

$$
\psi(T):=\xi^{-1}\left(\tilde{\varphi}\left(-T^{o}\right)\right) .
$$

It is clear that $\psi$ is a homomorphism of Lie algebras. Further, we have

$$
\varphi\left(T^{a} x\right)=\psi(T)^{\rho} \varphi(x)+\varphi(x) \psi(T) \quad \text { for } x \in V, T \in \mathfrak{m}_{0} .
$$

This follows from $\varphi\left(T^{o} x\right)=\tilde{\varphi}\left(T^{o}\right) \varphi(x)=R^{\rho} \varphi(x)+\varphi(x) R$ because

$$
R=-\xi^{-1}\left(\tilde{\varphi}\left(T^{o}\right)\right)=\psi(T) \quad \text { for } T \in \mathfrak{m}_{0} .
$$

We are now in the position to describe the essential part of $g_{0}$. For simplicity we write $(T, \hat{T})$ instead of $(z, u) \mapsto(T z, \hat{T} u)$ for the elements of $g_{0}$.

Lemma 3.10. The Lie algebra $\mathfrak{m}_{0}^{\varphi}:=\left\{(T, \psi(T)) ; T \in \mathfrak{m}_{0}\right\}$ is a subalgebra of $\mathfrak{g}_{0}$ and $\mathfrak{m}_{0}^{\circ}$ is the direct sum of the subspaces

$$
\begin{aligned}
& \mathfrak{m}_{i j}^{\varphi}:=\left\{\left(A_{e i}\left(x_{i j}\right), \frac{1}{2} \varphi\left(e_{j j}\right) \varphi\left(x_{i j}\right)\right) ; x_{i j} \in \mathfrak{H}_{i j}\right\} \quad \text { for } 1 \leq i<j \leq q . \\
& \mathfrak{m}_{i i}^{\varphi}:=\text { the Lie algebra generated by }\left\{\left(A\left(x_{i i}\right), \frac{1}{2} \varphi\left(x_{i i}\right)\right) ; x_{i i} \in \mathfrak{A}_{i i}\right\} ; \\
& 1 \leq i \leq q .
\end{aligned}
$$

Proof. A comparison with Lemma 2.10 shows $\mathfrak{m}_{0}^{\varphi} \subset \mathfrak{g}_{0}$. Further, it is clear that $\mathfrak{m}_{0}^{\varphi}$ is the direct sum of the subspaces $\mathfrak{m}_{i j}^{\varphi}:=\left\{(T, \psi(T)) ; T \in \mathfrak{m}_{i j}\right\}$. From (3.17) we get (3.20) and that $\mathfrak{m}_{i i}^{\varphi}$ is generated by $\left\{\left(A\left(x_{i i}\right), \frac{1}{2} \varphi\left(e_{i i}\right) \varphi\left(x_{i i}\right)\right)\right.$; $\left.x_{i i} \in \mathfrak{A}_{i i}\right\}$. Lemma 3.7 now proves the assertion.

Corollary 3.11. Let $i \leq j, x \in \mathfrak{A}_{i i}$ and $y \in \mathfrak{A}_{i j}$. Then

$$
\left(A_{x}(y), \frac{1}{2} \varphi(y) \varphi(x)\right) \in \mathrm{g}_{0} .
$$

Proof. From the lemma we know that

$$
\left(A(x), \frac{1}{2} \varphi(x)\right) \quad \text { and } \quad\left(A_{e i i}(y), \frac{1}{2} \varphi\left(e_{j j}\right) \varphi(y)\right)
$$

are elements of $g_{0}$. This implies that $(T, \hat{T}):=\left(A_{e_{i i}}(x y), \frac{1}{2} \varphi\left(e_{j j}\right) \varphi(x y)\right)+$ ( $\left.\left[A_{e_{i i}}(y), A(x)\right],\left[\frac{1}{2} \varphi\left(e_{j j}\right) \varphi(y), \frac{1}{2} \varphi(x)\right]\right)$ also lies in $g_{0}$. We use the "Grundformel" [33; Theorem 1.2] (see also [14; Theorem 1.2]) and get $T=A_{x}(y)$. It obviously suffices to prove $\varphi\left(e_{j j}\right) \varphi(x y)+\frac{1}{2} \varphi\left(e_{j j}\right) \varphi(y) \varphi(x)-\frac{1}{2} \varphi(x) \varphi\left(e_{j_{j}}\right) \varphi(y)=$ $\frac{1}{2} \varphi(y) \varphi(x)$. On $U_{i}$ and on $U_{j}$ this identity is easily verified; by Lemma 3.9 the assertion follows. To finish the preparations for the description of $g_{0}$ we set 


$$
\mathfrak{a}^{\varphi}:=\{(T, \hat{T}) ; T \in \mathfrak{a}, T S(u, w)=S(\hat{T} u, w)+S(u, \hat{T} w) \text { for } u, w \in U\} .
$$

Obviously $\mathfrak{a}^{\varphi} \subset \mathrm{g}_{0}$.

THEOREM 3.12. a) $\mathfrak{g}_{0}=\bigoplus_{1 \leq i \leq j \leq q} \mathfrak{m}_{i j}^{\varphi}+\mathfrak{a}^{\varphi}$ (direct sum of vector spaces).

b) The Lie algebras $\mathfrak{m}_{i i}^{\varphi}$ and $\mathfrak{a}^{\varphi}$ are selfadjoint with respect to $\sigma \oplus \rho$, hence they are reductive.

c) Split $\oplus_{i=1}^{q} \mathfrak{m}_{i i}^{\varphi}=\mathfrak{z}_{1}+\mathfrak{h}_{1}, \mathfrak{a}^{\varphi}=\mathfrak{z}_{2}+\mathfrak{h}_{2}$ into center $\mathfrak{z}_{j}$ and semisimple part $\mathfrak{h}_{j}$ and set $\mathfrak{n}^{\varphi}:=\bigoplus_{1 \leq i \leq j \leq q} \mathfrak{m}_{i j}^{\varphi}$, then

(1) $z_{1}+z_{2}$ is an abelian algebraic Lie algebra consisting of semisimple endomorphisms (direct sum of Lie algebras).

(2) $\mathfrak{h}_{1}+\mathfrak{h}_{2}$ is a maximal semisimple subalgebra of $\mathfrak{g}_{0}$ (direct sum of Lie algebras).

(3) $z_{1}+z_{2}+\mathfrak{n}^{\varphi}$ is the radical of $\mathfrak{g}_{0}$.

(4) $\mathfrak{n}^{\varphi}$ is the maximal ideal of $\mathrm{g}_{0}$ consisting of nilpotent endomorphisms.

Proof. a) Let $(T, \hat{T}) \in \mathfrak{g}_{0}$, then we know $T \in \operatorname{Lie} \operatorname{Aut}\left(K, \eta_{K S}\right)$. Therefore, by Theorem 3.1, there exist $T_{\mathfrak{m}} \in \mathfrak{m}_{0}$ and $T_{a} \in \mathfrak{a}$ such that $T=T_{\mathrm{m}}+$ $T_{\mathrm{a}}$. From Lemma 3.10 we get $\mathfrak{m}_{i j}^{\varphi} \subset \mathfrak{g}_{0}$; hence it exists a $\hat{T}_{\mathrm{m}} \in \operatorname{End}_{c} U$ satisfying $\left(T_{\mathrm{m}}, \hat{T}_{\mathrm{m}}\right) \in \mathfrak{g}_{0}$. This proves that $\mathfrak{g}_{0}$ is contained in the space at the right hand side of the formula. The converse inclusion has been proved above. It remains to show that the sum is direct. But $0=X_{\mathrm{m}}+$ $X_{\mathfrak{a}} \in \mathfrak{m}_{0}^{\varphi}+\mathfrak{a}^{\varphi}$ implies that the first components of $X_{\mathrm{m}}$ and $X_{\mathfrak{v}}$ are equal to 0 . Lemma 3.10 shows $X_{\mathrm{m}}=0$.

b) Clearly, $\left(A\left(x_{i i}\right), \frac{1}{2} \varphi\left(x_{i i}\right)\right)$ is selfadjoint with respect to $\sigma \oplus \rho$. Hence $\left(\mathfrak{m}_{i i}^{\varphi}\right)^{\sigma \oplus \rho} \subset \mathfrak{m}_{i i}^{\varphi}$. For $(T, \hat{T}) \in \mathfrak{a}^{\varphi}$ we have $T e=0$. By $[17 ;(6.11)]$ we get $T+\hat{T}=2 A(T e)=0$. From $0=\varphi\left(T^{\sigma} e\right)=\hat{T}^{\rho}+\hat{T}$ we derive that $(T, \hat{T})$ is skewadjoint with respect to $\sigma \oplus \rho$. Hence the assertion.

c) In b) we proved that all elements of $\mathfrak{a}^{\varphi}$ are skewadjoint with respect to $\sigma \oplus \rho$. Hence, for $(T, \hat{T}) \in \mathfrak{b}^{\varphi}$ we have $\left[\varphi\left(x_{i i}\right), \hat{T}\right]=\varphi\left(x_{i i}\right) \hat{T}+$ $\hat{T}^{\rho} \varphi\left(x_{i i}\right)=-\varphi\left(T x_{i i}\right)=0$ and $\left[A\left(x_{i i}\right), T\right]=A\left(T x_{i i}\right)=0$. This implies [ $\left.\mathfrak{m}_{i i}^{\varphi}, \mathfrak{a}^{\varphi}\right]$ $=0$. As a result we get $(1)$ and $\left[\mathfrak{h}_{1}, \mathfrak{h}_{2}\right]=0$. By a similar computation we see $\left[\mathfrak{n}^{\varphi}, \mathfrak{a}^{\varphi}\right] \subset \mathfrak{n}^{\varphi}$. It now suffices to prove that $\mathfrak{n}^{\varphi}$ is an ideal of $\mathfrak{g}_{0}$; for, $\mathfrak{n}^{\varphi}$ consists of nilpotent endomorphisms and $\mathfrak{n}^{\varphi}+z_{1}+z_{2}$ is contained in the radical of $g_{0}$; using this and a) we easily prove the assertion. It remains to show that $\mathfrak{n}^{\varphi}$ is an ideal of $g_{0}$. By the above remarks it suffices to verify $\left[\mathfrak{m}_{i i}^{\varphi}, \mathfrak{n}^{\varphi}\right] \subset \mathfrak{n}^{\varphi}$. But by $[15$; Theorem 7.3] we know that $\mathfrak{n}=$ $\oplus_{1 \leq i<j \leq q} \mathfrak{m}_{i j}$ is an ideal of $\mathfrak{m}$. Hence Lemma 3.10 implies the assertion.

Remark 3.13. a) Theorems 3.1 and 3.12 clarify the structure of homo- 
geneous Siegel domains. Our approach to the description of homogeneous Siegel domains uses larger "building blocks" than the approaches by $j$ algebras [43] or $S$-algebras [52]. This appears to have advantages for the description of the (infinitesimal) automorphisms. We get the building blocks of $S$-algebras when we form the Peirce decomposition with respect to a CSI $d_{1}, \cdots, d_{n}$-hence replacing $e_{11}, \cdots, e_{q q}$ by $d_{1}, \cdots, d_{n}$-which additionally satisfies $R d_{i}=\left\{x \in \mathfrak{U} ; d_{i} x=x\right\}, 1 \leq i \leq n$.

b) Examples show that the algebra $\mathfrak{a}^{\varphi}$ can be rather large. It is clear that $\mathfrak{a}^{\varphi}$ is contained in the Lie algebra of the isotropy group of the point ie.

For later use we mention some properties of $\mathfrak{a}^{\varphi}$.

LEMMA 3.14. a) $a^{\varphi}$ is the Lie algebra of a compact subgroup of Gl $D(K, S)$; hence $a^{\varphi}$ is reductive.

b) The elements of $\mathfrak{a}^{\varphi}$ are skewadjoint with respect to $\sigma \oplus \rho$.

c) $\mathfrak{a}^{\varphi}$ annihilates $\mathfrak{U}_{j j}$ and commutes with $\mathfrak{m}_{j j}^{\varphi}, 1 \leq j \leq q$.

d) $\mathfrak{a}^{\varphi}$ leaves invariant each of the spaces $\mathfrak{A}_{i j}, 1 \leq i, j \leq q$.

e) For each idempotent $b$ of $\mathfrak{X}=\oplus_{j=1}^{q} \mathfrak{A}_{j j}$ the space $\varphi(b) U$ is left invariant by $\mathfrak{a}^{\varphi}$.

Proof. a) To prove the first part of the assertion suffices to note that the isotropy subgroups of Aut $D(K, S)$ are compact and that $\mathrm{Gl} D(K, S)$ is closed in Aut $D(K, S)$. The second part now follows (see e.g. [9; IV, $\S 4$, Proposition 5]).

b) This has been shown in the proof of b) of Theorem 3.12.

c) By definition, $\mathfrak{a}^{\varphi}$ annihilates the spaces $\mathfrak{A}_{j j}$. The assertion follows from b) and Lemma 2.10.

d) Let $(T, \hat{T}) \in \mathfrak{a}^{\varphi}$. Then $T$ is a derivation of $\mathfrak{A}$ which annihilates all $e_{j j}$. Hence, $T$ commutes with all $A\left(e_{j j}\right)$. From (3.1) and (3.2) the assertion now easily follows.

e) Follows from b) and Lemma 2.10.

To finish this paragraph we consider the set

$$
\mathfrak{a}_{0}=\left\{(0, \hat{T}) \in \mathfrak{a}^{\varphi}\right\} .
$$

LEMMA 3.15. a) $\mathfrak{a}_{0}^{\varphi}$ is an ideal of $\mathfrak{g}_{0}$,

b) $\mathfrak{a}_{0}^{\varphi}=\left\{(0, \hat{T}) ; \hat{T}^{\rho}=-\hat{T}, \hat{T} \varphi(x)=\varphi(x) \hat{T}\right.$ for all $\left.x \in \mathfrak{A}\right\}$.

Proof. Part a) is obvious. To prove part b) we note that $0=S(\hat{T} u, w)$ $+S(u, \hat{T} w), u, w \in U$, is equivalent to $0=\sigma(x, S(\hat{T} u, w))+\sigma(x, S(u, \hat{T} w))$, 
$x \in V, u, w \in U$. Using (2.7) we see that this is equivalent to $0=\hat{T}^{\rho} \varphi(x)$ $+\varphi(x) \hat{T}, x \in V$. The assertion follows.

5. In this paragraph we consider a special infinitesimal automorphism of $K$. We use the decomposition $\mathfrak{U}=\mathfrak{U}_{1}+\mathfrak{U}_{\frac{1}{2}}+\mathfrak{U}_{0}$ of $\mathfrak{U}$ which has been introduced in 2. It is clear that this decomposition is a Peirce decomposition $\mathfrak{U}_{1}=\widetilde{\mathfrak{A}}_{11}, \mathfrak{A}_{\frac{1}{2}}=\widetilde{\mathfrak{A}}_{12}, \mathfrak{U}_{0}=\widetilde{\mathfrak{A}}_{22}$ of $\mathfrak{A}$ with respect to $d_{1}:=c_{1}=c, d_{2}:=$ $c_{0}$. Hence we may apply the results of the preceding sections.

The space $\varphi\left(c_{0}\right) U$ will be denoted by $U_{0}$ instead of $U_{2}$.

Lemma 3.16. The endomorphism $v \mapsto \operatorname{Re}(S(\varphi(v) u, w))$ of $V$ is an element of Lie Aut $\left(K, \eta_{K S}\right)$ for $u \in U_{1}$ and all $w \in U$.

Proof. Because of Lemma 2.2 we may apply [16; Theorems 1.7 and 3.3]. Hence it suffices to prove that $q(v, u, w):=\sigma(h(v), \operatorname{Re}(S(\varphi(v) u, w)))$, $v \in K$, does not depend on $v$. From [14; Theorem 1.8] we know that $v$ can be represented in the form $v=\exp A_{c}\left(v_{\frac{1}{2}}\right)\left(v_{1}+v_{0}\right)$ with $v_{\frac{1}{2}} \in \mathfrak{A}_{\frac{1}{2}}$ and $v_{i}$ $\in K_{i}, i=1,0$, appropriately chosen (for results on the projections $K_{i}$ of $K$ on $\mathfrak{A}_{i}$ we refer to [14].). From Lemma 3.10 we know $\left(A_{c}\left(v_{\frac{1}{2}}\right), \frac{1}{2} \varphi\left(c_{0}\right) \varphi\left(v_{\frac{1}{b}}\right)\right)$ $\in g_{0}$. Hence, for $W:=\exp A_{c}\left(v_{\frac{1}{2}}\right)$ and $\hat{W}:=\exp \frac{1}{2} \varphi\left(c_{0}\right) \varphi\left(v_{\frac{1}{2}}\right)$ we have the identities $h\left(W\left(v_{1}+v_{0}\right)\right)=W^{\sigma-1} h\left(v_{1}+v_{0}\right)$ and $W^{-1} S(y, z)=S\left(\hat{W}^{-1} y, \hat{W}^{-1} z\right)$ for all $y, z \in U$. It follows $q(v, u, w)=q\left(W\left(v_{1}+v_{0}\right), u, w\right)=\sigma\left(h\left(v_{1}+v_{0}\right), W^{-1}\right.$ $\operatorname{Re}(S(\varphi(v) u, w)))=\sigma\left(h\left(v_{1}+v_{0}\right), \operatorname{Re}\left(S\left(\hat{W}^{-1} \varphi\left(W\left(v_{1}+v_{0}\right)\right) u, \hat{W}^{-1} w\right)\right)\right)$. From $W\left(v_{1}+v_{0}\right)=v_{1}+v_{0}+v_{1} v_{\frac{1}{2}}+\frac{1}{2} c_{0}\left(v_{1} v_{\frac{1}{2}} \cdot v_{\frac{1}{2}}\right)$ we get by Lemma 3.9 the equality $\varphi\left(W\left(v_{1}+v_{0}\right)\right) u=\varphi\left(v_{1}+v_{1} v_{\frac{1}{2}}\right) u$. We use again Lemma 3.9 and get $\hat{W}^{-1}=$ $\exp -\frac{1}{2} \varphi\left(c_{0}\right) \varphi\left(v_{\frac{1}{2}}\right)=\operatorname{Id}-\frac{1}{2} \varphi\left(c_{0}\right)\left(v_{\frac{1}{2}}\right)$. Putting this together we have $q(v, u, w)$ $=\sigma\left(h\left(v_{1}+v_{0}\right), \operatorname{Re}(S(\mathrm{a}, \mathrm{b}))\right)$ with $a:=\varphi\left(v_{1}+v_{1} v_{\frac{1}{2}}\right) u-\frac{1}{2} \varphi\left(c_{0}\right) \varphi\left(v_{\frac{1}{2}}\right) \varphi\left(v_{1}+v_{1} v_{\frac{1}{2}}\right) u$ and $b:=w-\frac{1}{2} \varphi\left(c_{0}\right) \varphi\left(v_{\frac{1}{2}}\right) w$. From Corollary 3.8 and Lemma 3.9 we derive $\varphi\left(c_{0}\right) \varphi\left(v_{\frac{1}{2}}\right) \varphi\left(v_{1} v_{\frac{1}{2}}\right) u=0, \varphi\left(c_{0}\right) \varphi\left(v_{\frac{1}{2}}\right) w_{0}=0$, where $w=: w_{1}+w_{0}$ with $w_{j} \in U_{j}$, and $a=\varphi\left(v_{1}\right) u+\varphi\left(v_{1} v_{\frac{1}{2}}\right) u-\frac{1}{2} \varphi\left(v_{\frac{1}{2}}\right) \varphi\left(v_{1}\right) u$ and $b=w_{1}+w_{0}-\frac{1}{2} \varphi\left(v_{\frac{1}{2}}\right) w_{1}$. But by Lemma 3.6 and Lemma 3.9 we have $\varphi\left(v_{1} v_{1}\right) u-\frac{1}{2} \varphi\left(v_{\frac{1}{2}}\right) \varphi\left(v_{1}\right) u=0$ for $u \in U_{1}$. Hence $a=\varphi\left(v_{1}\right) u \in U_{1}$. Now we split $b=b_{1}+b_{0}$ with $b_{1}:=w_{1}$ and $b_{0}:=$ $w_{0}-\frac{1}{2} \varphi\left(v_{\frac{1}{2}}\right) w_{1}$. It follows $q(v, u, w)=\sigma\left(h\left(v_{1}+v_{0}\right), \operatorname{Re}\left(S\left(a, b_{1}\right)+S\left(a, b_{0}\right)\right)\right)=$ $q\left(v_{1}+v_{0}, u, w_{1}\right)+\sigma\left(h\left(v_{1}+v_{0}\right), \operatorname{Re}\left(S\left(a, b_{0}\right)\right)\right)$. We know $h\left(v_{1}+v_{0}\right) \in \mathfrak{A}_{1}+\mathfrak{U}_{0}$ and $\operatorname{Re}\left(S\left(a, b_{0}\right)\right) \in \mathfrak{A}_{\frac{1}{2}} ;$ Hence $q(v, u, w)=q\left(v_{1}+v_{0}, u, w_{1}\right)$. From [14; Lemma 2.2] we know $h\left(v_{1}+v_{0}\right)=h_{1}\left(v_{1}\right)+h_{0}\left(v_{0}\right)$ where $h_{j}\left(v_{j}\right) \in \mathfrak{A}_{j}, j=0,1$. We therefore get $q(v, u, w)=q\left(v_{1}, u, w_{1}\right)=\frac{1}{2} \rho\left(\varphi\left(h_{1}\left(v_{1}\right)\right) \varphi\left(v_{1}\right) u, w_{1}\right)+\frac{1}{2} \rho\left(\varphi\left(h_{1}\left(v_{1}\right)\right) w_{1}\right.$, $\left.\varphi\left(v_{1}\right) u\right)=\operatorname{Re}\left(\sigma\left(\varphi\left(h_{1}\left(v_{1}\right)\right) \varphi\left(v_{1}\right) u, w_{1}\right)\right)$. It obviously suffices to prove $\varphi\left(h_{1}\left(v_{1}\right)\right) \varphi\left(v_{1}\right)$ $=\varphi(c)$. But, by Lemma 3.6, the restriction of $\varphi$ to $\mathfrak{A}_{1}$ is a homomorphism 
of Jordan algebras and $h_{1}\left(v_{1}\right)$ is the inverse of $v_{1}$ in the Jordan algebra $\mathfrak{A}_{1}$ by [14; Corollary 2.5]. Hence the assertion.

Remark 3.17. a) If $w=w_{n} \in U_{0}$, then it is easy to verify directly $\operatorname{Re}\left(S\left(\varphi(v) u, w_{0}\right)\right)=A_{c}\left(\operatorname{Re}\left(S\left(u, w_{0}\right)\right)\right) v$. If $w=w_{1} \in U_{1}$ a similar representation is not known.

b) A statement which is weaker than that of Lemma 3.16 has been proved in [45].

6. We end this section with some remarks on nondegenerate Siegel domains. As usual a Siegel domain $D(K, S)$ is called nondegenerate if the set $\{S(u, w) ; u, w \in U\}$ generates the vector space $V^{c}$.

It is easy to see that $D(K, S)$ is nondegenerate if and only if the map $\varphi: \mathfrak{U} \rightarrow \operatorname{Sym}(U, \rho)$ is injective.

For the description of $\operatorname{Ker} \varphi$ we represent the Jordan algebra $\mathfrak{X}=$ $\left\{x \in V ; A(x) \in \operatorname{Lie} \operatorname{Aut}\left(K, \eta_{K S}\right)\right\}$ as the sum of its simple ideals $\mathfrak{X}_{i}, \mathfrak{X}=$ $\bigoplus_{i=1}^{m} \mathfrak{X}_{i}$. By $d_{i}$ we denote the unit of $\mathfrak{X}_{i}$ and form the Peirce decomposition $\mathfrak{U}=\oplus_{1 \leq i \leq j \leq m} \widetilde{\mathfrak{A}}_{i j}$ of $\mathfrak{X}$ with respect to $d_{1}, \cdots, d_{m}$.

In what follows we use without mentioning the following identity which was proved in Lemma 2.10:

$$
\varphi\left(T^{\sigma} x\right)=\hat{T}^{\rho} \varphi(x)+\varphi(x) \hat{T} \quad \text { for } x \in V \text { and }(T, \hat{T}) \in g_{0} .
$$

We obviously have

Lemma 3.18. $T^{\sigma} \operatorname{Ker} \varphi \subset \operatorname{Ker} \varphi$ for all $(T, \hat{T}) \in g_{0}$.

As a consequence of this fundamental property of $\operatorname{Ker} \varphi$ we get

Corollary 3.19. a) $\operatorname{Ker} \varphi=\oplus_{1 \leq i \leq j \leq m}\left(\overline{\mathfrak{A}}_{i j} \cap \operatorname{Ker} \varphi\right)$.

b) If $i<j$ and $\widetilde{\mathfrak{A}}_{i j} \subset \operatorname{Ker} \varphi$ then $\widetilde{\mathfrak{A}}_{i i} \subset \operatorname{Ker} \varphi$.

c) If $\widetilde{\mathfrak{A}}_{i i} \subset \operatorname{Ker} \varphi$ then $\widetilde{\mathfrak{A}}_{i j} \subset \operatorname{Ker} \varphi$ for all $1 \leq j \leq m$.

Proof. From $\left(A\left(d_{i}\right), \frac{1}{2} \varphi\left(d_{i}\right)\right) \in \mathfrak{g}_{0}$ we conclude that $A\left(d_{i}\right)$ leaves invariant $\operatorname{Ker} \varphi$ for all $1 \leq i \leq m$. This implies a) and c). To verify b) we use $\left(A_{d_{i}}\left(x_{i j}\right), \frac{1}{2} \varphi\left(x_{i j}\right) \varphi\left(d_{i}\right)\right) \in \mathfrak{g}_{0}$ and $\left[A_{d_{i}}\left(x_{i j}\right)\right]^{\sigma}=A_{d_{j}}\left(x_{i j}\right)$ for $x \in \widetilde{\mathfrak{A}}_{i j}$ and easily get the assertion.

Finally, we use again $\mathfrak{A}_{1}=\mathfrak{U}_{1}(c)=\widetilde{\mathfrak{A}}_{11}$ and get

Corollary 3.20. $\operatorname{Ker} \varphi \cap \mathfrak{U}_{1}($ resp. $\operatorname{Ker} \varphi \cap \mathfrak{X})$ is an ideal of $\mathfrak{N}_{1}$ (resp. $\mathfrak{X )}$ and $\operatorname{Ker} \varphi \neq 0$ implies $\operatorname{Ker} \varphi \cap \mathfrak{X} \neq 0$. 


\section{§4. Description of $\mathfrak{g}_{\frac{1}{2}}(K, S)$}

In this section we denote by $D(K, S)$ a homogeneous Siegel domain in $V^{C} \times U$.

We retain the notation of the preceding paragraphs.

By $P_{\frac{1}{2}}$ we denote the set of $w \in U$ which satisfy

$$
\begin{aligned}
& \varphi(c) w=w, \\
& S(\varphi(S(u, w)) u, d)=S(u, \varphi(S(d, u)) w) \quad \text { for all } u, d \in U .
\end{aligned}
$$

Theorem 4.1. $g_{\frac{1}{z}}=\left\{(z, u) \mapsto(2 S(u, \varphi(\bar{z}) w), i \varphi(z) w+2 \varphi(S(u, w)) u) ; w \in P_{\frac{1}{2}}\right\}$.

Proof. From Theorem 1.5 we derive that it suffices to show that a polynomial $X(z, u)=\left(p_{11}(z, u), q_{10}(z, u)+q_{02}(z, u)\right)$ can be represented as in the assertion if and only if it satisfies $\left[X, \mathfrak{g}_{-1}\right] \subset \mathfrak{g}_{-\frac{1}{2}}$ and $\left[X, \mathfrak{g}_{-\frac{1}{2}}\right] \subset \mathfrak{g}_{0}$.

To simplify notation we write $q_{10}(z)=q_{10}(z, u)$ and $q_{02}(u)=q_{02}(z, u)$.

As in [48] we first prove that $X \in \mathfrak{g}_{\frac{1}{2}}$ is equivalent to

(1) $p_{11}(z, u)=2 i S\left(u, q_{10}(\bar{z})\right)$,

(2) $2 S\left(u, q_{10}(S(\mathrm{~d}, u))\right)+i S\left(q_{02}(u), d\right)=0$,

(3) $\left(v \mapsto \operatorname{Im}\left(S\left(q_{10}(v), d\right)\right)\right) \in \operatorname{Lie} \operatorname{Aut}\left(K, \eta_{K S}\right)$ for all $z \in V^{c}, u, d \in U, v \in V$. A computation shows that $\left[X, \mathfrak{g}_{-1}\right] \subset \mathfrak{g}_{-\frac{1}{2}}$ is equivalent to (1). The condition $\left[X, g_{-\frac{1}{2}}\right] \subset \mathfrak{g}_{0}$ precisely means that $R:=\left(2 i S\left(q_{10}(z)+q_{02}(u), d\right)-\right.$ $\left.p_{11}(2 i S(u, d), u)-p_{11}(z, d),-q_{10}(2 i S(u, d))+q_{02}(u ; d)\right)$ is an element of $g_{0}$; here we put $q_{02}(u ; d):=\Delta_{u}^{d} q_{02}(u)$. Obviously, $R \in g_{0}$ is equivalent to

(a) $2 i S\left(q_{02}(u), d\right)-2 i p_{11}(S(u, d), u)=0$,

(b) $\quad\left((z, u) \mapsto\left(2 i S\left(q_{10}(z), d\right)-2 i S\left(d, q_{10}(z)\right),-2 i q_{10}(S(u, d))+q_{02}(u ; d)\right)\right) \in \mathfrak{g}_{0}$. Clearly, (a) is equivalent to (2) whereas (b) is equivalent to two conditions: (3) and

(c) $2 i S\left(q_{10}(S(u, w)), d\right)-2 i S\left(d, q_{10}(S(w, u))\right)=S\left(-2 i q_{10}(S(u, d))+\right.$ $\left.q_{02}(u ; d), w\right)+S\left(u,-2 i q_{10}\left(S(w, d)+q_{02}(w ; d)\right)\right.$.

We prove that (c) is a consequence of (2). We apply the operator $\Delta_{u}^{w}$ and get

(d) $2 S\left(w, q_{10}(S(d, u))\right)+2 S\left(u, q_{10}(S(d, w))\right)+i S\left(q_{02}(u ; w), d\right)=0$.

In this formula we replace the triple $(w, d, u) \in U \times U \times U$ by $(d, w, i u)$. It yields

(e) $2 i S\left(d, q_{10}(S(w, u))\right)+2 i S\left(\mathrm{u}, q_{10}(S(w, d))\right)-S\left(q_{02}(u ; d), w\right)=0$. Now, we conjugate $(\mathrm{d})$, replace $(w, d, u)$ by $(d,-i u, w)$ and get

(f) $-2 i S\left(q_{10}(S(u, w)), d\right)-2 i S\left(q_{10}(S(u, d)), w\right)-S\left(u, q_{02}(w ; d)\right)=0$. Adding (e) and (f) gives (c). This finishes the proof that $X \in g_{\frac{1}{2}}$ is equivalent to (1), (2) and (3). 
Next we exploit (3). By [16; Theorems 1.7 and 3.3] we know that a linear map $T: V \rightarrow V$ is an element of Lie Aut $\left(K, \eta_{K S}\right)$ if and only if $\sigma(h(v), T v), v \in K$, does not depend on $v$. Hence (3) is equivalent to

(3.a) $\sigma\left(h(v), S\left(q_{10}(v), d\right)\right)=\sigma\left(e, S\left(q_{10}(e), d\right)\right)$ for $v \in K$.

We set $w:=w_{1}+w_{0}:=q_{10}(e), w_{j} \in U_{j}$, use the definition of $\rho$, Lemma 2.9, and see that (3.a) is equivalent to

(3.b) $\varphi(h(v)) q_{10}(v)=w$ for all $v \in K$.

From Lemma 2.6 we know $h(v) \in K^{\sigma}$ for $v \in K$; hence, by Lemma 2.10, $\varphi(h(v))$ is invertible. This implies

(3.c) $q_{10}(v)=\varphi(h(v))^{-1} w$ for $v \in K$.

We differentiate this identity twice at $v=e$ and get

(4) $q_{10}(x)=\varphi(x) w, x \in V$,

(5) $\varphi(x y) w=\frac{1}{2}(\varphi(x) \varphi(y)+\varphi(y) \varphi(x)) w, x, y \in V$.

We use (4) to compute $q_{02}$ from (2) explicitly.

$$
\begin{aligned}
-i \rho\left(q_{02}(u), d\right) & =\sigma\left(e,-i S\left(q_{\mathrm{c} 2}(u), d\right)\right)=2 \sigma(e, S(u, \varphi(S(d, u)) w)) \\
& =2 \rho(u, \varphi(S(d, u)) w)=2 \rho(\varphi(S(u, d)) u, w) \\
& =2 \sigma(S(u, d), S(u, w))=2 \rho(\varphi(S(u, w)) u, d) .
\end{aligned}
$$

This implies

(6) $q_{02}(u)=2 i \varphi(S(u, w)) u$.

Using (4) and (6) it is easy to verify that $\mathfrak{g}_{z}$ consists exactly of those polynomials $(z, u) \mapsto(2 i S(u, \varphi(\bar{z}) w), \varphi(z) w+2 i \varphi(S(u, w)) u)$ where $w$ satisfies (4.2) and

(3.d) $(v \mapsto \operatorname{Im}(S(\varphi(v) w, d))) \in \operatorname{Lie} \operatorname{Aut}\left(K, \eta_{K S}\right)$.

Here we replace $w$ by $w_{1}+w_{0}$. Then, by Lemma 3.16, we see that (3.d) is equivalent to $T \in \operatorname{Lie} \operatorname{Aut}\left(K, \eta_{K S}\right)$ where $T$ is defined by

$$
T v:=\operatorname{Im}\left(S\left(\varphi(v) w_{0}, d\right)\right) \text {. }
$$

Because of Theorem 3.3 we may apply [14; Theorem 3.3] and thus get $x_{1}$ $\in \mathfrak{A}_{1}, x_{\frac{1}{2}} \in \mathfrak{A}_{\frac{1}{2}}$ and a derivation $D$ of the algebra $\mathfrak{A}_{c}$ which satisfies $D c=0$, such that $T=A_{c}\left(x_{1}\right)+A_{c}\left(x_{\frac{1}{2}}\right)+D$. From the definition of $T$ we immediately derive $T \mathfrak{N}_{1}=0$. Hence $x_{1}=0$ and $x_{\frac{1}{2}}=0$. This means that $T$ is a derivation of $\mathfrak{U}_{c}$ which leaves invariant the spaces $\mathfrak{U}_{i}$. But from the definition of $T$ we get $T \mathfrak{Y}_{1} \subset \mathfrak{Y}_{1}$ and $T \mathfrak{Y}_{0} \subset \mathfrak{U}_{\frac{1}{2}}$. Hence $T=0$. This especially implies $\varphi\left(v_{\frac{1}{2}}\right) w_{0}=0$. We use (5) and get $\varphi\left(c_{0} v_{\frac{1}{2}}^{2}\right) w_{0}=0$ for all $v_{\frac{1}{2}} \in \mathfrak{U}_{\frac{1}{2}}$. Theorem 3.4 now shows $w_{0}=0$. The theorem is proved.

Corollary 4.2. Each $X \in \mathfrak{g}_{\frac{1}{2}}$ is uniquely determined by its value at ie. 
Corollary 4.3. For $w \in U_{1}$ the following are equivalent

(1) $w \in p_{\frac{1}{2}}$,

(2) $S(\varphi(S(u, w)) u, d)=S(u, \varphi(S(d, u)) w)$ for all $u, d \in U$,

(3) The map $(z, u) \mapsto(S(\varphi(z) w, d)+S(d, \varphi(\bar{z}) w),-\varphi(S(u, d)) w+$ $\varphi(S(u, w)) d+\varphi(S(d, w)) u)$ is for all $d \in U$ an element of $g_{0}$.

Proof. $\quad(1) \Leftrightarrow(2)$ : clear by definition of $P_{\frac{1}{2}}$.

(2) $\Rightarrow(3)$ : insert $q_{10}(z)=i \varphi(z) w$ and $q_{02}(u)=2 \varphi(S(u, w)) u$ in the statement (b) of the proof of Theorem 4.1.

$(3) \Rightarrow(2)$ : by assumption we have $S(\varphi(S(a, b)) w, d)+S(d, \varphi(S(b, a)) w)$ $=S(-\varphi(S(a, d)) w+\varphi(S(a, w)) d+\varphi(S(d, w)) a, b)+S(a,-\varphi(S(b, d)) w+$ $\varphi(S(b, w)) d+\varphi(S(d, w)) b)$ for all $a, b, d \in U$.

We replace the tuple $(a, w, d, b)$ by $(u, w, u, d)$ and get

(a) $S(\varphi(S(u, d)) w, u)+S(u, \varphi(S(d, u)) w)=S(-\varphi(S(u, u)) w, d)+$ $S(\varphi(S(u, w)) u, d)+S(\varphi(S(u, w)) u, d)+S(u,-\varphi(S(d, u)) w)+$ $S(u, \varphi(S(d, w)) u)+S(u, \varphi(S(u, w)) d)$ for $u, d \in U$.

Now we replace $(a, w, d, b)$ by $(u, w, d, u)$. It results

(b) $S(\varphi(S(u, u)) w, d)+S(d, \varphi(S(u, u)) w)=-S(\varphi(S(u, d)) w, u)+$ $S(\varphi(S(u, w)) d, u)+S(\varphi(S(d, u)) u, u)-S(u, \varphi(S(u, d)) w)+S(u, \varphi(S(u, w)) d)+$ $S(u, \varphi(S(d, w)) u)$ for $u, d \in U$.

From (b) we derive

(c) $\operatorname{Re}(S(\varphi(S(u, u)) w, d))=-\operatorname{Re}(S(\varphi(S(u, d)) w, u))+$ $\operatorname{Re}(S(u, \varphi(S(u, w)) d))+\operatorname{Re}(S(u, \varphi(S(d, u)) u))$ for all $u, d \in U$. This implies

(d) $S(\varphi(S(u, u)) w, d)=-S(\varphi(S(u, d)) w, u)+S(u, \varphi(S(u, w)) d)+$ $S(u, \varphi(S(d, u)) u)$ for all $u, d \in U$.

A comparison of (a) with (d) gives (2).

Remark 4.4. a) In [48] Theorem 4.1 has been proven under the additional assumption that $D(K, S)$ is symmetric.

b) For symmetric Siegel domains we always have $\mathfrak{A}_{\frac{1}{2}}=0$ and $\mathfrak{A}_{0}=$ 0 . With this additional assumption the proof of Theorem 4.1 simplifies considerably.

c) $P_{\frac{1}{2}}$ is a vector space over $\boldsymbol{C}$ but $g_{\frac{1}{2}}$ is only a vector space over $\boldsymbol{R}$.

d) Corollary 4.3 has been proved in [48] for symmetric Siegel domains. Finally, we expand (4.2) in terms of $U_{1}$ and $U_{0}$.

LEMMA 4.5. Let $w_{1} \in U_{1}$; then (4.2) is equivalent to the following set of equations (where $u_{1}, d_{1} \in U_{1}$ and $u_{0}, d_{0} \in U_{0}$ are arbitrary 

a) $S\left(\varphi\left(S\left(u_{1}, w_{1}\right)\right) u_{1}, d_{1}\right)=S\left(u_{1}, \varphi\left(S\left(d_{1}, u_{1}\right)\right) w_{1}\right)$
b) $0=\varphi\left(S\left(u_{0}, w_{1}\right)\right) u_{0}$,
c) $S\left(\varphi\left(S\left(u_{1}, w_{1}\right)\right) u_{1}, d_{0}\right)=S\left(u_{1}, \varphi\left(S\left(d_{0}, u_{1}\right)\right) w_{1}\right)$,
d) $0=S\left(u_{1}, \varphi\left(S\left(d_{1}, u_{0}\right)\right) w_{1}\right)+S\left(u_{1}, \varphi\left(S\left(w_{1}, u_{0}\right)\right) d_{1}\right)$.

Proof. In (4.2) we replace $u$ by $u_{1}+u_{0}$ and $d$ by $d_{1}+d_{0}$. Then we expand (4.2) and compare in $\mathfrak{A}_{k}+i \mathfrak{Y}_{k}, k=1, \frac{1}{2}, 0$. This yields three equations. We use the fact that $u_{j}$ and $d_{j}$ can be chosen arbitrary and get the following set of equations which is equivalent to (4.2).

(1) $S\left(\varphi\left(S\left(u_{1}, w_{1}\right)\right) u_{1}, d_{1}\right)=S\left(u_{1}, \varphi\left(S\left(d_{1}, u_{1}\right)\right) w_{1}\right)$,

(2) $S\left(\varphi\left(S\left(u_{0}, w_{1}\right)\right) u_{0}, d_{1}\right)=0$,

(3) $S\left(\varphi\left(S\left(u_{0}, w_{1}\right)\right) u_{1}, d_{1}\right)=S\left(u_{1}, \varphi\left(S\left(d_{1}, u_{0}\right)\right) w_{1}\right)+S\left(u_{0}, \varphi\left(S\left(d_{1}, u_{1}\right)\right) w_{1}\right)$,

(4) $S\left(\varphi\left(S\left(u_{1}, w_{1}\right)\right) u_{1}, d_{0}\right)=S\left(u_{1}, \varphi\left(S\left(d_{0}, u_{1}\right)\right) w_{1}\right)$,

(5) $S\left(\varphi\left(S\left(u_{0}, w_{1}\right)\right) u_{0}, d_{0}\right)=0$,

(6) $S\left(\varphi\left(S\left(u_{0}, w_{1}\right)\right) u_{1}, d_{0}\right)=S\left(u_{0}, \varphi\left(S\left(d_{0}, u_{1}\right)\right) w_{1}\right)$,

(7) $S\left(u_{0}, \varphi\left(S\left(d_{1}, u_{r}\right)\right) w_{1}\right)=0$.

Clearly, (1) and a) coincide as do (4) and c). It is easy to verify that (2) and (5) together are equivalent to $b$ ). Hence it remains to investigate (3), (6) and (7). Here we use that $\left(A_{c}\left(x_{\frac{1}{2}}\right), \frac{1}{2} \varphi\left(c_{0}\right) \varphi\left(x_{\frac{1}{2}}\right)\right)$ and $\left(A\left(x_{1}\right), \frac{1}{2} \varphi\left(x_{1}\right)\right)$ are elements of $\mathfrak{g}_{0}$ for all $x_{1} \in \mathfrak{U}_{1}, x_{\frac{1}{2}} \in \mathfrak{U}_{\frac{1}{2}}$ i.e. we have the following identities at our disposal

$$
\begin{aligned}
& 2 A_{c}\left(x_{\frac{1}{2}}\right) S(a, b)=S\left(\varphi\left(c_{0}\right) \varphi\left(x_{\frac{1}{2}}\right) a, b\right)+S\left(a, \varphi\left(c_{0}\right) \varphi\left(x_{\frac{1}{2}}\right) b\right) \text {, } \\
& 2 A\left(x_{1}\right) S(a, b)=S\left(\varphi\left(x_{1}\right) a, b\right)+S\left(a, \varphi\left(x_{1}\right) b\right) \\
& \text { for all } x_{k} \in \mathfrak{A}_{k}, k=1, \frac{1}{2} \text { and all } a, b \in U \text {. }
\end{aligned}
$$

By $(*)$ and $(* *)$ we get from (3) equivalently

$$
\begin{aligned}
& S\left(\varphi\left(S\left(u_{0}, w_{1}\right)\right) u_{1}, d_{1}\right)-S\left(u_{1}, \varphi\left(S\left(d_{1}, u_{0}\right)\right) w_{1}\right)=S\left(u_{0}, \varphi\left(S\left(d_{1}, u_{1}\right)\right) w_{1}\right) \\
& \quad=2 A\left(S\left(u_{1}, d_{1}\right)\right) S\left(u_{0}, w_{1}\right)=2 A_{c}\left(S\left(u_{0}, w_{1}\right)\right) S\left(u_{1}, d_{1}\right) \\
& \left.\quad=S\left(\varphi\left(S\left(u_{0}, w_{1}\right)\right) u_{1}, d_{1}\right)+S\left(u_{1}, \varphi\left(S\left(w_{1}, u_{0}\right)\right) d_{1}\right) \text { hence } d\right)
\end{aligned}
$$

In $(*)$ we put $x_{\frac{1}{2}}:=S\left(u_{0}, w_{1}\right)$; then (6) easily follows. It remains to show that (7) is a consequence of a) to d). To prove (7) it suffices to show that the left hand side of (7) is orthogonal to all $\mathfrak{A}_{i j}, 2 \leq i \leq j \leq q$. Thus let $x_{i j} \in \mathfrak{A}_{i j}$ and $(T, \hat{T}) \in \mathfrak{m}_{i j}^{\varphi}, 2 \leq i \leq j \leq q$, such that $T^{\sigma} e=T e=x_{i j}$ holds. Then $\hat{T} U_{1}=0$ and $T$ is a derivation of the algebra $\mathfrak{H}_{c}[14 ; \S 3]$. We use this, $(*)$ and $b$ ) and get 


$$
\begin{aligned}
& \sigma\left(S\left(u_{0}, \varphi\left(S\left(d_{1}, u_{0}\right)\right) w_{1}\right), x_{i j}\right)=2 \sigma\left(T A_{c}\left(S\left(u_{0}, d_{1}\right)\right) S\left(u_{0}, w_{1}\right), e\right) \\
&= \sigma\left(S\left(\hat{T} u_{0}, d_{1}\right)+S\left(u_{0}, \hat{T} d_{1}\right), S\left(u_{0}, w_{1}\right)\right)+\sigma\left(S\left(u_{0}, d_{1}\right), S\left(\hat{T} u_{0}, w_{1}\right)\right. \\
&\left.\quad+S\left(u_{0}, \hat{T} w_{1}\right)\right)=\sigma\left(S\left(\hat{T} u_{0}, d_{1}\right), S\left(u_{0}, w_{1}\right)\right)+\sigma\left(S\left(u_{0}, d_{1}\right), S\left(\hat{T} u_{0}, w_{1}\right)\right) \\
&= \rho\left(\varphi\left(S\left(u_{0}, w_{1}\right)\right) \hat{T} u_{0}+\varphi\left(S\left(\hat{T} u_{0}, w_{1}\right)\right) u_{0}, d_{1}\right)=0 .
\end{aligned}
$$

This finishes the proof.

\section{§5. Description of $\mathfrak{g}_{1}(K, S)$}

In this section we finish the description of Lie Aut $D(K, S)$. By $D(K, S)$ we denote a homogeneous Siegel domain in $V^{c} \times U$. We use again the decomposition $\mathfrak{U}=\mathfrak{U}_{1}+\mathfrak{U}_{\frac{1}{2}}+\mathfrak{X}_{0}$ of $\mathfrak{U}$ which has been introduced in $\S 3,2$. The unit of $\mathfrak{X}_{1}$ is denoted by $c=c_{1}=e_{11}$ and we set $U_{1}=\varphi(c) U$ and $U_{0}=\varphi\left(c_{0}\right) U$ where $c_{0}=e-c$. We then have $U=U_{1}+U_{0}$.

Finally, we mention that in this section we frequently use mutations of $\mathfrak{A}$ which have been introduced in $\S 2,2$.

We set

$$
\begin{aligned}
P_{1}:= & \left\{x \in \mathfrak{A}_{1} ; \text { it exists } B \in \operatorname{End}_{C} U \text { such that }(z, u) \mapsto\left(A_{x}(z) z, \varphi(z) B u\right)\right. \\
& \text { is an element of } \left.g_{1}\right\} .
\end{aligned}
$$

Lemma 5.1. Let $(T, \hat{T}) \in \mathfrak{g}_{0} ;$ then

$$
T^{\sigma} P_{1} \subset P_{1} \quad \text { and } \quad \hat{T}^{\rho} P_{\frac{1}{2}} \subset P_{\frac{1}{2}} .
$$

Proof. Let $x \in P_{1}$ and $X$ the associated element of $\mathfrak{g}_{1}$. We form the commutator of $X$ and $(T, \hat{T})$ and get $T A_{x}(z) z-2 A_{x}(z) T z$ as first component. The "Grundformel" [33; Theorem 1.2] implies that this expression equals $-A_{T} \sigma_{x}(z) z$. Because of $[X,(T, \hat{T})] \in g_{1}$ the first assertion follows. To prove the second one we choose $w \in P_{\frac{1}{2}}$ and denote by $Y$ the associated element of $g_{\frac{1}{2}}$. Then the linear part of the second component of $[Y,(T, \hat{T})]$ equals $T i \varphi(v) w-i \varphi(T v) w$. From this we derive that $[Y,(T, \hat{T})] \in \mathfrak{g}_{\frac{1}{2}}$ is parametrized by $T i \varphi(e) w-i \varphi(T e) w=-i \hat{T}^{\rho} w$ where we have used Lemma 2.10.

We specialize $(T, \hat{T}):=\left(A(y), \frac{1}{2} \varphi(y)\right), y \in \mathfrak{A}_{1}$, and get

Corollary 5.2. $P_{1}$ is an ideal of $\mathfrak{U}_{1}$.

By the corollary, we know in particular that $P_{1}$ is a subalgebra of the formally real Jordan algebra $\mathfrak{N}_{1}$ and hence has a unit which we denote by $p$ or $p_{1}$. We sometimes abbreviate $p_{0}:=e-p$.

Corollary 5.3. Let $X$ be the element of $g_{1}$ which is associated to $p \in$ $P_{1}$. Then we have $(\operatorname{ad} X)^{2} \mathfrak{g}_{-1}=\mathfrak{g}_{1}$ and $(\operatorname{ad} X) \mathfrak{g}_{0}=\mathfrak{g}_{1}$. 
Proof. By a straightforward computation we see that the first component of $(\operatorname{ad} X)^{2} Y, Y:=(a, 0) \in \mathfrak{g}_{-1}$, equals $r(z):=-2 A_{p}(a) A_{p}(z) z+$ $4 A_{p}\left(A_{p}(\mathrm{a}) z\right) z$. Because of $A_{p}(v) \in \operatorname{Lie} \operatorname{Aut}\left(K, \eta_{K S}\right)$ for $v \in V$, the "Grundformel" [33; Theorem 1.2] implies $r(z)=A_{b}(z) z$ where $b=2\left[A_{p}(a)\right]^{\sigma} e=$ $A(p a)^{\sigma} e=p a$. The first assertion follows. The second one is proved in an analogous way.

The first part of the last corollary has also been proved in [45].

Theorem 5.4. $P_{1}=\left\{x \in \mathfrak{A}_{1} ;(z, u) \mapsto\left(A_{x}(z) z, \varphi(z) \varphi(x) u\right) \in \mathfrak{g}_{1}\right\}$.

$$
\mathfrak{g}_{1}=\left\{(z, u) \mapsto\left(A_{x}(z) z, \varphi(z) \varphi(x) u\right) ; x \in P_{1}\right\} .
$$

Proof. By Theorem 1.5 we know that each $X \in g_{1}$ can be represented in the form $X(z, u)=\left(p_{20}(z), q_{11}(z, u)\right)$. We prove

(1) $X(z, u)=\left(A_{x}(z) z, \varphi(z) B u\right)$ where $x \in \mathfrak{A}_{1}$ and $B \in \operatorname{End}_{C} U$. We mention that the polynomial $p_{20}$ is contained in the Lie algebra $g(K)$ of the group of automorphisms of the tube domain $V+i K$ by [29; Theorem 4]. Let $\widetilde{\mathfrak{A}}$ denote the algebra which is constructed for $D(K)=V+i K$ and $e \in K$ according to $\S 2,2$. The left multiplications of this algebra are denoted by $\tilde{A}(v), v \in V$. From Theorem 2.8 we know $p_{20}(z)=\tilde{A}_{a}(z) z$ for all $z \in V^{c}$ and $a \in \tilde{\mathfrak{A}}$ appropriate. Now, we represent $q_{11}$ in the form $q_{11}(z, u)=\tilde{B}(z) u$ where $\tilde{B}$ is a $C$-linear map from $V^{c}$ into $\operatorname{End}_{\boldsymbol{c}} U$. From $\left[X, g_{-1}\right] \subset g_{0}$ we conclude $\left(\tilde{A}_{a}(v), \frac{1}{2} \tilde{B}(v)\right) \in g_{0}$ for all $v \in V$. We apply Theorem 3.3 and [33; Theorem 5.10] to get an $x \in \mathfrak{A}_{1}$ such that $\tilde{A}_{a}(v)=A_{x}(v)$ holds for all $v \in V$. Finally, we choose $Y(z, u)=(2 i S(u, d), d) \in \mathfrak{g}_{-\frac{1}{2}}$ and compute $[X, Y] \in \mathfrak{g}_{\frac{1}{2}}$. The linear term of the second component of $[X, Y]$ turns out to be $\tilde{B}(z) d$. Whence, by Theorem 4.1 we get a $w \in P_{\frac{1}{z}}$ such that $\tilde{B}(z) d=i \varphi(z) w$ holds for all $z \in V^{c}$. We set $B:=\tilde{B}(e)$ and it results $\tilde{B}(z) d=\varphi(z) B d$ for all $z \in V^{c}$. This proves (1). Next we claim

(2) A polynomial $X(z, u)=\left(A_{x}(z) z, \varphi(z) B u\right), x \in \mathfrak{A}_{1}, \mathrm{~B} \in \operatorname{End}_{c} U$, is an element of $g_{1}$ if and only if

(2.a) $\left(A_{x}(a), \frac{1}{2} \varphi(a) B\right) \in \mathfrak{g}_{0}$ for all $a \in V$,

(2.b) $\operatorname{Im}(\operatorname{trace} \varphi(a) B)=0$ for all $a \in V$,

(2.c) $B U \subset P_{\frac{1}{2}}$,

(2.d) $\varphi(S(u, d)) B u=\varphi(S(u, B d)) u$ for all $u, d \in U$.

We apply part b) of Theorem 1.5. Clearly, (1.23) is equivalent to (2.a) and (1.25) is equivalent to (2.b). Finally, choose $Y \in g_{-\frac{1}{2}}, Y(z, u)=$ $(2 i S(u, d), d)$ and compute

$$
[X, Y]=\left(2 i S(\varphi(z) B u, d)-4 i A_{x}(z) S(u, d),-2 i \varphi(S(u, d)) B u-\varphi(z) B d\right) .
$$


We use (2.a) and see that the first component of this expression equals $-2 i S(u, \varphi(\bar{z}) B d)$. Because of $[X, Y] \in \mathfrak{g}_{\frac{1}{2}}$ we get from Theorem 4.1 that $w:=i B d$ is an element of $P_{\frac{1}{2}}$. Comparing the expression for $[X, Y]$ which we have computed above with the expression for the element of $g_{\frac{1}{2}}$ which is associated to $w$ it is easy to verify that (1.24) and (2.a) are equivalent to (2.a), (2.c) and (2.d). This proves (2). In the next step of the proof we compute $\left[\mathfrak{g}_{\frac{1}{2}}, \mathfrak{g}_{\frac{1}{2}}\right]$.

(3) Let $w, w^{\prime} \in P_{\frac{1}{2}}$ and $Y, Y^{\prime}$ the corresponding elements of $g_{\frac{1}{2}}$. Set $y:=-4 \operatorname{Im}\left(S\left(w, w^{\prime}\right)\right)$; then $\left[Y, Y^{\prime}\right](z, u)=\left(A_{y}(z) z, \varphi(z) \varphi(y) u\right)$ for all $(z, u) \epsilon$ $V^{c} \times U$.

Because of $\left[Y, Y^{\prime}\right] \in \mathfrak{g}_{1}$ we know from (1) that there exists a $y \in \mathfrak{A}_{1}$ and a $B \in \operatorname{End}_{C} U$ such that $\left[Y, Y^{\prime}\right](z, u)=\left(A_{y}(z) z, \varphi(z) B u\right)$ holds. A straightforward computation of $\left[Y, Y^{\prime}\right]$ produces

(3.a) $A_{y}(z) z=2 S\left(i \varphi(z) w, \varphi(\bar{z}) w^{\prime}\right)-2 S\left(i \varphi(z) w^{\prime}, \varphi(\bar{z}) w\right)$,

(3.b) $\varphi(z) B u=i \varphi(2 S(u, \varphi(\bar{z}) w)) w^{\prime}+2 \varphi\left(S\left(i \varphi(z) w, w^{\prime}\right)\right) u+$ $2 \varphi\left(S\left(u, w^{\prime}\right)\right) i \varphi(z) w-i \varphi\left(2 S\left(u, \varphi(\bar{z}) w^{\prime}\right)\right) w-2 \varphi\left(S\left(i \varphi(z) w^{\prime}, w\right)\right) u-$ $2 \varphi(S(u, w)) i \varphi(z) w^{\prime}$.

From these two equations we obviously get $y=-4 \operatorname{Im}\left(S\left(w, w^{\prime}\right)\right)$ and $B=\varphi(y)$.

(4) $y \in P_{1}, \varphi(y) P_{\frac{1}{2}}=0 \Rightarrow \varphi(y)=0$.

Consider the map $\kappa: \mathfrak{U}_{1} \rightarrow \operatorname{Sym}\left(P_{\frac{1}{2}}, \rho\right),\left.y \mapsto \varphi(y)\right|_{P_{\frac{1}{2}}}$. It is well defined because of Lemma 5.1 and it is a homomorphism of Jordan algebras because of Lemma 3.6. Hence, Ker $\kappa$ is an ideal of $\mathfrak{A}_{1}$. By Corollary 5.2 also Ker $\kappa$ $\cap P_{1}$ is an ideal of the formally real Jordan algebra $\mathfrak{A}_{1}$. This implies that $\operatorname{Ker} \kappa \cap P_{1}$ has a unit; we denote it by $r$. Let $Y \in \mathfrak{g}_{1}$ such that $Y(z, u)$ $=\left(A_{r}(z) z, \varphi(z) B u\right)$ holds. From (2.a) and Corollary 3.11 we derive that it exists $C \in \operatorname{End}_{C} U$ which satisfies $B=\varphi(r)+C$ and $(0, \varphi(a) C) \in g_{0}$ for all $a \in V$. Put $a:=e$ then Lemma 2.10 implies $C^{\rho}=-C$ and $[\varphi(v), C]=0$ for all $v \in V$. Consequently $[\varphi(r), B]=0$ and by (2.c) we get $0=\varphi(r) B U$ $=B \varphi(r) U$. From $\rho\left(B^{\rho} \varphi(r) u, w\right)=\rho(u, \varphi(r) B w)=\rho(u, B \varphi(r) w)=0, u, w \in U$, we conclude that the restrictions of $B$ and $B^{\rho}$ vanish on $\varphi(r) U$. Then also $B+B^{\rho}=\varphi(r)$ vanishes on $\varphi(r) U$. Whence $\varphi(r)=0$. Let $y \in \operatorname{Ker} \kappa$ $\cap P_{1}$ be arbitrary; then $\varphi(y)=\varphi(r y)=\frac{1}{2}\{\varphi(r) \varphi(y)+\varphi(y) \varphi(r)\}=0$. This proves (4).

Set $P_{1}^{(0)}:=\operatorname{Ker} \varphi \cap P_{1}$. As in the proof of (4) we see that $P_{1}^{(0)}$ is an ideal of the formally real Jordan algebra $P_{1}$. Hence there exists an ideal $P_{1}^{(1)}$ of $P_{1}$ such that $P_{1}=P_{1}^{(0)} \oplus P_{1}^{(1)}$ holds. 
(5) $\left\{S(w, w) ; w \in P_{\frac{1}{2}}\right\}$ generates the vector space $P_{1}^{(1)}$.

From (3) we know $S\left(P_{\frac{1}{2}}, P_{\frac{1}{2}}\right) \subset P_{1}+i P_{1}$. Denote by $r$ the unit element of $P_{1}^{(0)}$. Then $(A(r), 0) \in \mathfrak{g}_{n}$ whence $r S\left(w, w^{\prime}\right)=0$ for all $w, w^{\prime} \in P_{\frac{1}{2}}$. This implies $S\left(P_{\frac{1}{2}}, P_{\frac{1}{2}}\right) \subset P_{1}^{(1)}+i P_{1}^{(1)}$. On the other hand let $x \in P_{1}^{(1)}$ such that $\sigma(x, S(w, w))=0$ for all $w \in P_{\frac{1}{2}}$. Consequently $\rho(\varphi(x) w, w)=0$; so we get $\varphi(x) w=0$ for all $w \in P_{\frac{1}{2}}$. From (4) it follows $x \in \operatorname{Ker} \varphi \cap P_{1}^{(1)}=0$.

(6) The polynomial $(z, u) \mapsto\left(A_{x}(z) z, \varphi(z) \varphi(x) u\right)$ is for all $x \in P_{1}$ an element of $\mathfrak{g}_{1}$.

Let $x \in P_{1}^{(0)}$; we have to prove that the $\operatorname{map}(z, u) \mapsto\left(A_{x}(z), 0\right)$ is an element of $g_{1}$. From (2) we derive that only $\left(A_{x}(a), 0\right) \in \mathfrak{g}_{0}$ must be checked. But this is a consequence of Corollary 3.11.

Let $x \in P_{1}^{(1)}$; then the assertion follows from (3) and (5). This finishes the proof of (6).

To prove the theorem it now obviously suffices to show that $g_{1}$ contains no nontrivial map of the form $(z, u) \mapsto(0, B(z) u)$. If such a map is contained in $\mathfrak{g}_{1}$ then $(0, B(a)) \in \mathfrak{g}_{0}$ for all $a \in V$. This implies $B(a)^{\rho}=-B(a)$. On the other hand we have $0=\left[\mathfrak{g}_{1},\left[\mathfrak{g}_{1}, \mathfrak{A}_{-\frac{1}{2}}\right]\right]$; by a computation we derive from this $B(z)^{2}=0$. Consequently $B(z)=0$ for all $z \in V^{c}$. The theorem is proved.

Corollary 5.5. $\operatorname{Ker} \varphi \cap \mathfrak{A}_{1} \subset P_{1}$. For tube domains $\mathfrak{A}_{1}=P_{1}$ holds.

Proof. We use (2) from the proof of Theorem 5.4 and see that we only have to check $\left(A_{x}(a), 0\right) \in \mathfrak{g}_{0}$ for all $x \in \operatorname{Ker} \varphi \cap \mathfrak{U}_{1}$ and all $a \in V$. But this is clear by Corollary 3.11 .

More precisely we have

Corollary 5.6. The vector space $P_{1}^{(1)}$ which is spanned by $\{S(w, w)$; $\left.w \in P_{\frac{1}{2}}\right\}$ is an ideal of $P_{1}$ and we have

$$
P_{1}=\left(\operatorname{Ker} \varphi \cap \mathfrak{A}_{1}\right) \oplus P_{1}^{(1)} \text {. }
$$

Proof. Clear from Corollary 5.5 and (5) from the proof of Theorem 5.4. The last result implies

Corollary 5.7. a) If $P_{1} \not \subset \operatorname{Ker} \varphi$ then $\mathrm{g}_{\frac{1}{2}} \neq 0$.

b) If $\mathfrak{g}_{\frac{1}{2}} \neq 0$ then $\mathfrak{g}_{1} \neq 0$.

The next result characterizes $P_{1}$ without recourse to $g_{1}$.

CoRollary 5.8. For $x \in \mathfrak{U}_{1}$ the following are equivalent

(1) $x \in P_{1}$, 
(2) $\varphi(x) U \subset P_{\frac{1}{2}}$,

(3) $S(\varphi(S(u, \varphi(x) w)) u, d)=S(u, \varphi(S(d, u)) \varphi(x) w)$ for all $d, u, w \in U$.

Proof. Because of $x \in \mathfrak{X}_{1}$ and (4.2) it is clear that (2) is equivalent to (3). To prove (1) $\Rightarrow(2)$ we start with $x \in P_{1}$; then, by Theorem 5.4 , the map $(z, u) \mapsto\left(A_{x}(z) z, \varphi(z) \varphi(x) u\right)$ is an element of $g_{1}$. Hence we may apply (2.c) from the proof of Theorem 5.4 and get (2). Assume now that (2) holds. By Corollary 5.2 we know that $P_{1}$ is an ideal of the formally real Jordan algebra $\mathfrak{N}_{1}$. Hence there exists an ideal $\tilde{P}_{1}$ of $\mathfrak{N}_{1}$ satisfying $\mathfrak{U}_{1}=$ $P_{1} \oplus \tilde{P}_{1}$. The unit of $\tilde{P}_{1}$ is denoted by $g$. To prove (1) it clearly suffices to show $g x=0$. To verify this we first mention that by (2) and Lemma 5.1 we have $\varphi(g x) U=\frac{1}{2}\{\varphi(g) \varphi(x)+\varphi(x) \varphi(g)\} U \subset \varphi(g) \varphi(x) U+\varphi(x) U \subset \varphi(g) P_{\frac{1}{2}}$ $+P_{\frac{1}{2}} \subset P_{\frac{1}{2}}$. By (3) from the proof of Theorem 5.4 we get $y:=S(\varphi(g x) u$, $\varphi(g x) u)=-\operatorname{Im}(S(\varphi(g x) u, \varphi(g x) i u)) \in P_{1}$. Because of $\varphi(p) \varphi(g x)=0$ we have $p y=0$. But $y \in P_{1}$, whence $y=0$. From (1.12) we get $\varphi(g x) u=0$ for all $u \in U$. Therefore, $g x \in \operatorname{Ker} \varphi \cap \mathfrak{X}_{1}$. Corollary 5.5 now implies $g x \in P_{1}$. Hence $g x=0$. The corollary is proved.

Corollary 5.9. $\varphi(p) U=P_{\frac{1}{2}}$.

Proof. Because of (2) of Corollary 5.8 we only have to prove $P_{\frac{1}{2}} \subset$ $\varphi(p) U$. Let $w \in P_{\frac{1}{2}}$; as $\varphi(p) U \subset P_{\frac{1}{2}}$ we may assume $\varphi(p) w=0$. From Corollary 5.6 we know $S(w, w) \in P_{1}$, whence $S(w, w)=p S(w, w)=$ $\frac{1}{2} S(\varphi(p) w, w)+\frac{1}{2} S(w, \varphi(p) w)=0$. This implies $w=0$ and the assertion is proved.

As a trivial consequence of the last result we get

Corollary 5.10. Let $X$ denote the element of $\mathfrak{g}_{1}$ which is associated to $p \in P_{1}$. Then $(\operatorname{ad} X) g_{-\frac{1}{2}}=g_{\frac{1}{2}}$.

A similar result has been directly proved in [45]. We are now able to simplify the conditions of Theorem 1.5.

Corollary 5.11. For $X \in \tilde{\mathfrak{g}}_{1}$ the following are equivalent

(1) $X \in g_{1}$,

(2) $\left[X, \mathfrak{g}_{-1}\right] \subset \mathfrak{g}_{0}$ and $\left[X, \mathfrak{g}_{-\frac{1}{2}}\right] \subset \mathfrak{g}_{\frac{1}{2}}$.

Proof. Obviously, we only have to prove (2) $\Rightarrow(1)$. Let $X \in \tilde{\mathfrak{g}}_{1}$ satisfy the conditions of (2). From $\left[X, \mathfrak{g}_{-1}\right] \subset \mathfrak{g}_{0}$ we conclude that the first component of $X$ lies in $g_{1}(K) \subset$ Lie Aut $D(K)$. As in (1) from the proof of 
Theorem 5.4 we show $X(z, u)=\left(A_{x}(z) z, q_{11}(z, u)\right)$. We also derive $q_{11}(z, u)$ $=\varphi(z) B u$ from $\left[X, g_{-\frac{1}{2}}\right] \subset g_{\frac{1}{2}}$. Checking the proof of (2) from the proof of Theorem 5.4 we see that the conditions $\left[X, \mathfrak{g}_{-1}\right] \subset g_{0}$ and $\left[X, \mathfrak{g}_{-\frac{1}{2}}\right] \subset \mathfrak{g}_{\frac{1}{2}}$ are equivalent to (2.a), (2.c) and (2.d). It now suffices to note that the condition $\operatorname{Im}(\operatorname{trace} \varphi(a) B)=0$ for $a \in V$ was not used in the proof of Theorem 5.4 .

From Theorem 5.4 we get further

CoRollary 5.12. The elements of $\mathfrak{g}_{1}$ are uniquely determined by their value at ie.

Corollary 5.13. For $x \in \mathfrak{X}_{1}$ the following are equivalent

(1) $\varphi(S(u, w)) \varphi(x) u=\varphi(S(u, \varphi(x) w)) u$ for all $u, w \in U$,

(2) $\varphi(x) \varphi(S(u, w)) u=\varphi(S(\varphi(x) u, w)) u$ for all $u, w \in U$.

For $x \in P_{1}$ the conditions (1) and (2) are satisfied.

Proof. The first assertion follows from

$$
\begin{gathered}
\varphi(S(u, w)) \varphi(x) u-\varphi(S(u, \varphi(x) w)) u=2 \varphi(x S(u, w)) u-\varphi(x) \varphi(S(u, w)) u \\
-\varphi(S(u, \varphi(x) w)) u=\varphi(S(\varphi(x) u, w)) u-\varphi(x) \varphi(S(u, w)) u
\end{gathered}
$$

The second assertion follows from (2.d) from the proof of Theorem 5.4.

Remark 5.14. Theorem 5.4 has been proved for symmetric Siegel domains in [48]. The proofs are different.

We finish this section with a detailed description of $P_{1}$ and $P_{\frac{1}{2}}$. Let $\mathfrak{A}_{1}=\mathfrak{U}_{1}^{(1)} \oplus \ldots \oplus \mathfrak{U}_{1}^{(s)}$ be a decomposition of $\mathfrak{U}_{1}$ as sum of ideals. We denote the unit of $\mathfrak{R}_{1}^{(j)}$ by $c^{(j)}$. Then, by Lemma 3.9, $U_{1}$ is the orthogonal sum of the spaces $U_{1}^{(j)}:=\varphi\left(c^{(j)}\right) U_{1}$. Let $\widetilde{\mathfrak{A}}_{i j}$ denote the Peirce spaces of the Peirce decomposition of $\mathfrak{U}$ with respect to the CSI $f_{1}:=c^{(1)}, \cdots, f_{s}:=c^{(s)}$, $f_{s+1}:=c_{0}$; then $\mathfrak{U}_{1}=\bigoplus_{1 \leq i \leq j \leq s} \widetilde{\mathfrak{A}}_{i j}, \widetilde{\mathfrak{A}}_{j j}=\mathfrak{U}_{1}^{(j)}, \mathfrak{U}_{\frac{1}{2}}=\oplus_{1 \leq j \leq s} \widetilde{\mathfrak{A}}_{j, s+1}$ and $\mathfrak{U}_{0}=$ $\widetilde{\mathfrak{A}}_{s+1, s+1}$. The choice of $c^{(j)}$ implies that $A\left(c^{(j)}\right)$ has only the eigenvalues 0 and 1 on $\mathfrak{A}_{1}$ whence $\widetilde{\mathfrak{A}}_{i j}=0$ for $1 \leq i, j \leq s, i \neq j$. For such $i$ and $j$ we have $\widetilde{\mathfrak{A}}_{j, s+1} \widetilde{\mathfrak{A}}_{i, s+1} \subset \widetilde{\mathfrak{A}}_{i j}=0$. Further, from Lemma 3.9 we get $S\left(U_{1}^{(i)}, U_{1}^{(j)}\right)$ $\subset \widetilde{\mathfrak{A}}_{i j}=0$.

The following result is a very useful detailed description of $P_{1}$ and $P_{\frac{1}{2}}$.

Lemma 5.15. Assume that $\widetilde{\mathfrak{A}}_{1}^{(j)}$ is simple for all $j$. Then

a) $P_{\frac{1}{2}}=\bigoplus_{j=1}^{s}\left(P_{\frac{1}{2}} \cap U_{1}^{(j)}\right)$,

b) $P_{1}=\oplus_{j=1}^{s}\left(P_{1} \cap \mathfrak{Q}_{1}^{(j)}\right)$, 
c) If $P_{\frac{1}{2}} \cap U_{1}^{(j)} \neq 0$ then $P_{\frac{1}{2}} \cap U_{1}^{(j)}=U_{1}^{(j)}$ and $P_{1} \cap \mathfrak{Q}_{1}^{(j)}=\mathfrak{A}_{1}^{(j)}$,

d) If $P_{1} \cap \mathfrak{A}_{1}^{(j)} \neq 0$ then $P_{1} \cap \mathfrak{Q}_{1}^{(j)}=\mathfrak{N}_{1}^{(j)}$ and $P_{\frac{1}{2}} \cap U_{1}^{(j)}=U_{1}^{(j)}$.

Proof. By Corollary 5.2, $P_{1}$ is an ideal of $\mathfrak{U}_{1}$, hence sum of some $\mathfrak{N}_{1}^{(j)}$. We may assume $P_{1}=\mathfrak{U}_{1}^{(1)} \oplus \cdots \oplus \mathfrak{U}_{1}^{(k)}$. From Corollary 5.7 we get $\oplus_{j=1}^{k} U_{1}^{(j)}$ $=P_{\frac{1}{2}}$. Hence a), b) and the first parts of c) and d) are proved. To prove the second part of $c$ ) we choose $y \in P_{\frac{1}{2}} \cap U_{1}^{(j)}, y \neq 0$; then $S(y, y) \in P_{1}$. From $y \neq 0$ we get $S(y, y) \neq 0$; hence $P_{1} \cap \mathfrak{A}_{1}^{(j)} \neq 0$. By assumption, $\mathfrak{A}_{1}^{(j)}$ is simple, whence $P_{1} \cap \mathfrak{Q}_{1}^{(j)}=\mathfrak{U}_{1}^{(j)}$. Finally, the assumption of d) implies $\varphi\left(c^{(j)}\right) U=U_{1}^{(j)} \subset P_{\frac{1}{2}}$, whence the assertion.

Remark 5.16. Let $K_{1}^{(j)}$ denote the projection of $K$ onto $\mathfrak{A}_{1}^{(j)}$ and define $S_{j}: U_{1}^{(j)} \times U_{1}^{(j)} \rightarrow \mathfrak{U}_{1}^{(j)}+i \mathfrak{U}_{1}^{(j)}$ by $S_{j}(u, w):=S(u, w)$. Assume further that $\mathfrak{O}_{1}^{(j)}$ is simple. Then it can be shown that $D\left(K_{1}^{(j)}, S_{j}\right)$ is symmetric if and only if $P_{1} \cap \mathfrak{Q}_{1}^{(j)} \neq 0$.

\section{§6. Commutators in $\mathrm{g}(K, S)$}

In this section we express the commutators in $\mathfrak{g}(K, S)$ by parameters.

By $D(K, S)$ we denote a homogeneous Siegel domain in $V^{c} \times U$. We first fix some notation.

$$
P_{-1}:=V, \quad P_{-\frac{1}{2}}:=U, \quad P_{0}:=g_{0} .
$$

By Theorem 1.4 we know that $P_{-1}$ is a domain of parameters of $g_{-1}$ and that $U$ is a domain of parameters for $g_{-\frac{1}{2}}$.

We recall that $P_{\frac{1}{2}}$ and $P_{1}$ have been defined in the preceding sections as the respective domain of parameters for $g_{\frac{1}{2}}$ and $g_{1}$. It is clear that all $P_{\lambda}$ are vector spaces over $R$. $P_{-\frac{1}{2}}$ and $P_{\frac{1}{2}}$ are vector spaces over $\boldsymbol{C}$.

Let $\lambda \neq 0$ and $w \in P_{\lambda}$; then by $X_{\lambda}[w]$ we denote the element of $\mathfrak{g}_{\lambda}$ which is characterized by $w$ (see Theorems 1.4, 4.1 and 5.4). To unify notation we also put $X_{0}[T, \hat{T}]:=(T, \hat{T})$ for $(T, \hat{T}) \in \mathfrak{g}_{0}$.

For the description of all commutators it clearly suffices to consider the commmutators in $\left[\mathfrak{g}_{\lambda}, \mathfrak{g}_{\mu}\right] \subset \mathfrak{g}_{\lambda+\mu}$. Here we proceed as follows. At one hand we directly compute $L\left(w, w^{\prime}\right):=\left[X_{\lambda}[w], X_{\mu}\left[w^{\prime}\right]\right]$; on the other hand we know $L\left(w, w^{\prime}\right) \in \mathfrak{g}_{\lambda+\mu}$. Hence $L\left(w, w^{\prime}\right)=0$ if $\lambda+\mu \notin\left\{-1,-\frac{1}{2}, 0, \frac{1}{2}, 1\right\}$ and there exists an element $f\left(w, w^{\prime}\right) \in P_{\lambda+\mu}$ such that

$$
L\left(w, w^{\prime}\right)=X_{\lambda+\mu}\left[f\left(w, w^{\prime}\right)\right] \quad \text { holds if } \lambda+\mu \in\left\{-1,-\frac{1}{2}, 0, \frac{1}{2}, 1\right\} .
$$

In what follows we evaluate this equation case by case. More precisely, 
we compute $f\left(w, w^{\prime}\right)$ in all cases and derive several identities which turn out to be helpful in concrete computations.

All parameters appearing are chosen arbitrarily. The proofs, being easy, are omitted.

$$
\begin{aligned}
& {\left[X_{-1}[w], X_{-1}\left[w^{\prime}\right]\right]=0} \\
& {\left[X_{-1}[w], X_{-\frac{1}{2}}\left[w^{\prime}\right]\right]=0} \\
& {\left[X_{-1}[w], X_{0}[T, \hat{T}]\right]=X_{-1}[T w] .} \\
& {\left[X_{-1}[w], X_{\frac{1}{2}}\left[w^{\prime}\right]\right]=X_{-\frac{1}{2}}\left[i \varphi(w) w^{\prime}\right] .} \\
& {\left[X_{-1}[w], X_{1}\left[w^{\prime}\right]\right]=X_{0}\left[2 A_{w^{\prime}}(w), \varphi(w) \varphi\left(w^{\prime}\right)\right]} \\
& {\left[X_{-\frac{1}{2}}[w], X_{-\frac{1}{2}}\left[w^{\prime}\right]\right]=X_{-1}\left[-4 \operatorname{Im}\left(S\left(w, w^{\prime}\right)\right)\right]} \\
& {\left[X_{-\frac{1}{2}}[w], X_{0}[T, \hat{T}]\right]=X_{-\frac{1}{2}}[\hat{T} w] .} \\
& {\left[X_{-\frac{1}{2}}[w], X_{\frac{1}{2}}\left[w^{\prime}\right]\right](z, u)=2\left(S\left(w, \varphi(\bar{z}) w^{\prime}\right)+S\left(\varphi(z) w^{\prime}, w\right),\right.} \\
& \left.\quad-\varphi(S(u, w)) w^{\prime}+\varphi\left(S\left(w, w^{\prime}\right)\right) u+\varphi\left(S\left(u, w^{\prime}\right)\right) w\right)
\end{aligned}
$$

(6.10) $\quad\left[X_{-\frac{1}{2}}[w], X_{1}\left[w^{\prime}\right]\right]=X_{\frac{1}{2}}\left[-i \varphi\left(w^{\prime}\right) w\right]$.

(6.10.a) $\varphi(S(u, w)) \varphi\left(w^{\prime}\right) u=\varphi\left(S\left(u, \varphi\left(w^{\prime}\right) w\right)\right) u \quad$ for $u, w \in U, w^{\prime} \in P_{1}$.

(6.11) $\quad\left[X_{0}[T, \hat{T}], X_{0}\left[T^{\prime}, \hat{T}^{\prime}\right]\right]=X_{0}\left[-\left[T, T^{\prime}\right],-\left[\hat{T}, \hat{T}^{\prime}\right]\right]$.

(6.12) $\quad\left[X_{0}[T, \hat{T}], X_{\frac{1}{2}}[w]\right]=X_{\frac{1}{2}}\left[\hat{T}^{\rho} w\right]$.

The following three identities are valid for all $z \in V^{c}, u \in U, w \in P_{\frac{1}{2}},(T, \hat{T})$ $\in \mathfrak{g}_{0}$.

(6.12.a) $\varphi(T z) w-\hat{T} \varphi(z) w=\varphi(z) \hat{T}^{\rho} w$,

(6.12.b) $S(u, \varphi(T \bar{z}) w)-S(u, \hat{T} \varphi(\bar{z}) w)=S\left(u, \varphi(\bar{z}) \hat{T}^{\rho} w\right)$,

(6.12.c) $\varphi(S(\hat{T} u, w)) u+\varphi(S(u, w)) \hat{T} u-\hat{T} \varphi(S(u, w)) u=\varphi\left(S\left(u, \hat{T}^{\circ} w\right)\right) u$.

(6.13) $\quad\left[X_{0}[T, \hat{T}], X_{1}[w]\right]=X_{1}\left[T^{\sigma} w\right]$.

$$
\begin{aligned}
& \varphi(T z) \varphi(w)+\varphi(z) \varphi(w) \hat{T}-\hat{T} \varphi(z) \varphi(w)=\varphi(z) \varphi\left(T^{\sigma} w\right) \\
& \qquad \text { for all } z \in V^{c}, w \in P_{1},(T, \hat{T}) \in \mathfrak{g}_{0} .
\end{aligned}
$$

(6.14) $\quad\left[X_{\frac{1}{2}}[w], X_{\frac{1}{2}}\left[w^{\prime}\right]\right]=X_{1}\left[-4 \operatorname{Im}\left(S\left(w, w^{\prime}\right)\right)\right]$.

The next four identities hold for all $u \in U, z \in V^{c}, w, w^{\prime} \in P_{\frac{1}{2}}$.

$$
\begin{aligned}
& S\left(u, \varphi(S(\varphi(\bar{z}) w, u)) w^{\prime}\right)+S\left(\varphi(S(u, w)) u, \varphi(\bar{z}) w^{\prime}\right) \\
& \quad=S\left(u, \varphi\left(S\left(\varphi(\bar{z}) w^{\prime}, u\right)\right) w\right)+S\left(\varphi\left(S\left(u, w^{\prime}\right)\right) u, \varphi(\bar{z}) w\right),
\end{aligned}
$$

(6.14.b) $\varphi\left(S\left(u, w^{\prime}\right)\right) \varphi(S(u, w)) u=\varphi(S(u, w)) \varphi\left(S\left(u, w^{\prime}\right)\right) u$, 
(6.14.c) $-2 A_{\operatorname{Im}\left(S\left(w, w^{\prime}\right)\right)}(z) z=i S\left(\varphi(z) w, \varphi(\bar{z}) w^{\prime}\right)-i S\left(\varphi(z) w^{\prime}, \varphi(\bar{z}) w\right)$,

$$
\begin{aligned}
& -2 \varphi(z) \varphi\left(\operatorname{Im}\left(S\left(w, w^{\prime}\right)\right)\right) u=i \varphi(S(u, \varphi(\bar{z}) w)) w^{\prime}+i \varphi\left(S\left(\varphi(z) w, w^{\prime}\right)\right) u \\
& \quad+i \varphi\left(S\left(u, w^{\prime}\right)\right) \varphi(z) w-i \varphi\left(S\left(u, \varphi(\bar{z}) w^{\prime}\right)\right) w-i \varphi\left(S\left(\varphi(z) w^{\prime}, w\right)\right) u \\
& \quad-i \varphi(S(u, w)) \varphi(z) w^{\prime} .
\end{aligned}
$$

$$
\left[X_{\frac{1}{2}}[w], X_{1}\left[w^{\prime}\right]\right]=0 \text {. }
$$

The following three identities hold for all $w \in P_{\frac{1}{2}}, w^{\prime} \in P_{1}, u \in U, z \in V^{c}$.

$$
\begin{aligned}
& 2 A_{w^{\prime}}(z) S(u, \varphi(\bar{z}) w)=S\left(\varphi(z) \varphi\left(w^{\prime}\right) u, \varphi(\bar{z}) w\right)+S\left(u, \varphi\left(A_{w^{\prime}}(\bar{z}) \bar{z}\right) w\right) . \\
& \varphi(S(u, \varphi(\bar{z}) w)) \varphi\left(w^{\prime}\right) u+\varphi(z) \varphi\left(w^{\prime}\right) \varphi(S(u, w)) u \\
& \quad=\varphi\left(S\left(\varphi(z) \varphi\left(w^{\prime}\right) u, w\right)\right) u+\varphi(S(u, w)) \varphi(z) \varphi\left(w^{\prime}\right) u .
\end{aligned}
$$

(6.15.c) $\varphi\left(A_{w^{\prime}}(z) z\right) w=\varphi(z) \varphi\left(w^{\prime}\right) \varphi(z) w$.

(6.16) $\left[X_{1}[w], X_{1}\left[w^{\prime}\right]\right]=0$.

The last two identities are valid for all $w, w^{\prime} \in P_{1}, u \in U, z \in V^{c}$.

(6.16.a) $\quad A_{w^{\prime}}(z) A_{w}(z) z=A_{w}(z) A_{w^{\prime}}(z) z$.

$$
\begin{aligned}
& \varphi(z) \varphi\left(w^{\prime}\right) \varphi(z) \varphi(w) u+\varphi\left(A_{w}(z) z\right) \varphi\left(w^{\prime}\right) u \\
& \quad=\varphi(z) \varphi(w) \varphi(z) \varphi\left(w^{\prime}\right) u+\varphi\left(A_{w^{\prime}}(z) z\right) \varphi(w) u
\end{aligned}
$$

\section{§7. The radical and a Levi decomposition of $\mathfrak{g}(K, S)$}

Let $D(K, S)$ denote a homogeneous Siegel domain in $V^{c} \times U$. We use the results of the preceding sections to describe the radical and a Levi decomposition of $g(K, S)$. Finally, we associate to $D(K, S)$ in a canonical way a symmetric Siegel domain which turns out to be a concrete realization of a symmetric domain which has been abstractly constructed by K. Nakajima in [38].

1. To describe the radical $\mathfrak{w}=\mathfrak{w}(K, S)$ of $\mathfrak{g}=\mathfrak{g}(K, S)$ we use Theorem 1.6. But first we have to fix some notation.

$$
\begin{aligned}
& P_{-1}^{r}:=\left\{x \in V ; X_{-1}[x] \in \mathfrak{w}_{-\frac{1}{1}}\right\}, \\
& P_{-\frac{1}{2}}^{r}:=\left\{d \in U ; X_{-\frac{1}{2}}[d] \in \mathfrak{w}_{-\frac{1}{2}}\right\}, \\
& P_{1}^{\perp}:=\left\{x \in V ; \sigma(x, y)=0 \text { for all } y \in P_{1}\right\}, \\
& P_{\frac{1}{2}}^{\perp}:=\left\{d \in U ; \rho(d, u)=0 \text { for all } u \in P_{\frac{1}{2}}\right\} .
\end{aligned}
$$

THEOREM 7.1. a) $P_{-1}^{r}=P_{1}^{\perp}$,

b) $P_{-\frac{1}{2}}^{r}=P_{\frac{1}{2}}^{\perp}$ 
Proof. By a dimension argument we derive from Theorem 1.6 that it suffices to prove $P_{-1}^{r} \subset P_{1}^{\perp}$ and $P_{-\frac{1}{2}}^{r} \subset P_{\frac{1}{2}}^{\perp}$. Let $y \in P_{-1}^{r}$; then $X_{-1}[y] \in \mathfrak{w}_{-1}$ and for all $X_{1}\left[w^{\prime}\right] \in \mathfrak{g}_{1}$ we get $\left[X_{-1}[y], X_{1}\left[w^{\prime}\right]\right] \in \mathfrak{w} \cap \mathfrak{g}_{0} \subset \mathfrak{w}_{0}$. We know $\mathfrak{w} \cap$ $\mathrm{g}_{1}=0$; hence $\left[\left[X_{-1}[y], X_{1}\left[w^{\prime}\right]\right], X_{1}\left[w^{\prime}\right]\right]=0$. Now we use (6.6) and (6.13) and get $0=\left[A_{y}\left(w^{\prime}\right)\right]^{\sigma} w^{\prime}=A_{w^{\prime}}(y) w^{\prime}$ for all $w^{\prime} \in P_{1}, y \in P_{-1}^{r}$. We insert for $w^{\prime}$ the unit $p$ of the ideal $P_{1}$ of $\mathfrak{A}_{1}$. We thus get $0=A_{p}(y) p=p y$. This implies $y \in P_{1}^{\perp}$ and a) is proved. Now, let $d \in P_{-\frac{1}{2}}^{r}$, then we have $\left[X_{-\frac{1}{2}}[d]\right.$, $\left.X_{-\frac{1}{2}}\left[w^{\prime}\right]\right] \in \mathfrak{w} \cap \mathfrak{g}_{-1}=\mathfrak{w}_{-1}$ for all $w^{\prime} \in U, d \in P_{-\frac{1}{2}}^{r}$. Applying (6.7) and a) we get $\operatorname{Im}\left(S\left(d, w^{\prime}\right)\right) \in P_{1}^{\perp}$. We specialize $w^{\prime}:=\mathrm{id}$; hence $S(d, d) \in P_{1}^{\perp}$. By (6.8) we may replace $d$ by $\varphi(p) d$; in this case we have $0=\sigma(p, S(\varphi(p) d, \varphi(p) d))$ $=\rho(\varphi(p) d, \varphi(p) d)$, whence $0=\varphi(p) d$. But this means $d \in P_{\frac{1}{2}}^{\perp}$. The theorem is proved.

Now we characterize $\mathfrak{w}_{0}$.

Theorem 7.2. Let $X=(T, \hat{T}) \in g_{0}$. Then the following are equivalent.

(1) $X \in \mathfrak{w}_{0}$,

(2) $X \in \operatorname{rad} \mathfrak{g}_{0},\left[X, \mathfrak{g}_{-1}\right] \subset \mathfrak{w}_{-1},\left[X, \mathfrak{g}_{-\frac{1}{2}}\right] \subset \mathfrak{w}_{-\frac{1}{2}}$,

(3) $X \in \operatorname{rad} g_{0}, T^{o} P_{1}=0, \hat{T}^{\circ} P_{\frac{1}{2}}=0$.

Proof. Let $\widetilde{\mathfrak{w}}_{0}$ denote the set of $X$ 's which satisfy (2) and $\widetilde{\mathfrak{w}}_{0}$ the set of $X$ 's which satisfy (3). We obviously have $\mathfrak{w}_{0} \subset \widetilde{\mathfrak{w}}_{0}$. To prove $\widetilde{\mathfrak{w}}_{0}=\widetilde{\mathfrak{w}}_{0}$ let $X=(T, \hat{T}) \in \operatorname{rad} g_{0}$; then $X \in \tilde{\mathfrak{w}}_{0}$ is equivalent to $T V \subset P_{1}^{\perp}$ and $\hat{T} U \subset$ $P_{\frac{1}{2}}^{\perp}$ because of (6.4) and (6.8). Hence $X \in \widetilde{\mathfrak{w}}_{0}$ if and only if $T^{\circ} P_{1}=0$ and $\hat{T}^{\rho} P_{\frac{1}{2}}=0$. This proves $\tilde{\mathfrak{w}}_{0}=\tilde{\mathfrak{w}}_{0}$. To prove the theorem it suffices to show that $\mathfrak{c}:=\mathfrak{w}_{-1}+\mathfrak{w}_{-\frac{1}{2}}+\widetilde{\mathfrak{w}}_{0}$ is a solvable ideal of $\mathfrak{g}$. We first note

(a) $\left[\mathfrak{c}, \mathfrak{g}_{1}\right] \subset \mathfrak{c},\left[\mathfrak{c}, \mathfrak{g}_{-\frac{1}{2}}\right] \subset \mathfrak{c}$,

for $\left[\mathfrak{c}, \mathfrak{g}_{-1}\right] \subset\left[\tilde{\mathfrak{w}}_{0}, \mathfrak{g}_{-1}\right] \subset \mathfrak{w}_{-1}$ by (6.2) and (6.3).

(b) $\left[c, g_{1}\right] \subset \mathfrak{c},\left[c, g_{2}\right] \subset c$.

By the properties of $\mathfrak{g}$ it is clear that $\left[\mathfrak{c}, \mathfrak{g}_{1}\right] \subset \mathfrak{w}_{0}+\left[\tilde{\mathfrak{w}}_{0}, \mathfrak{g}_{1}\right]$ holds. It obviously suffices to prove $\left[\tilde{\mathfrak{w}}_{0}, \mathfrak{g}_{1}\right]=0$. But this follows from (6.13) and $T^{\circ} P_{1}$ $=0$ for $(T, \hat{T}) \subset \tilde{\mathfrak{w}}_{0}$. The second assertion is proved along the same lines using $\left[\mathfrak{c}, \mathfrak{g}_{\frac{1}{2}}\right] \subset \mathfrak{w}_{-\frac{1}{2}}+\mathfrak{w}_{0}+\left[\tilde{\mathfrak{w}}_{0}, \mathfrak{g}_{\frac{1}{2}}\right]$ and (6.12). Next we verify

(c) $\left[c, g_{0}\right] \subset \widetilde{\mathfrak{w}}_{0}$.

Because of $\left[\mathfrak{c}, \mathfrak{g}_{0}\right] \subset \mathfrak{w}_{-1}+\mathfrak{w}_{-\frac{1}{2}}+\left[\tilde{\mathfrak{w}}_{0}, \mathfrak{g}_{0}\right]$ it suffices to show $\left[\widetilde{\mathfrak{w}}_{0}, \mathfrak{g}_{0}\right] \subset \tilde{\mathfrak{w}}_{0}$. By (2) this means $\left[\tilde{\mathfrak{w}}_{0}, \mathfrak{g}_{0}\right] \subset \mathrm{rad} \mathfrak{g}_{0},\left[\left[\tilde{\mathfrak{w}}_{0}, \mathfrak{g}_{0}\right], \mathfrak{g}_{-1}\right] \subset \mathfrak{w}_{-1}$ and $\left[\left[\tilde{\mathfrak{w}}_{0}, \mathfrak{g}_{0}\right], \mathfrak{g}_{-\frac{1}{2}}\right] \subset \mathfrak{w}_{-\frac{1}{2}}$. Here the first inclusion is clear. Let $X \in \tilde{\mathfrak{w}}_{0}, Y \in \mathfrak{g}_{0}$ and $Z_{\nu} \in \mathfrak{g}_{\nu}, \nu=-1,-\frac{1}{2}$. We then have $\left[[X, Y], Z_{\nu}\right]=\left[X,\left[Y, Z_{\nu}\right]\right]+\left[Y,\left[Z_{\nu}, X\right]\right]$, and by definition of $\widetilde{\mathfrak{w}}_{0},\left[Z_{\nu}, X\right]$ is contained in $\mathfrak{w}_{\nu}$. Hence $\left[Y,\left[Z_{\nu}, X\right]\right] \in \mathfrak{w}_{\nu} \subset \mathfrak{c}, \nu=-1,-\frac{1}{2}$. On the other hand $\left[Y, Z_{\nu}\right] \subset \mathfrak{g}_{\nu}$; whence $\left[X,\left[Y, Z_{\nu}\right]\right] \in \mathfrak{w}_{\nu} \subset \mathcal{c}$. It now remains 
to prove that $\mathfrak{c}$ is solvable. Here we use $[\mathfrak{c}, \mathfrak{c}] \subset\left[\mathfrak{w}_{-1}, \tilde{\mathfrak{w}}_{0}\right]+\left[\mathfrak{w}_{-\frac{1}{2}}, \mathfrak{w}_{-\frac{1}{2}}\right]+$ $\left[\mathfrak{w}_{-\frac{1}{2}}, \widetilde{\mathfrak{w}}_{0}\right]+\left[\tilde{\mathfrak{w}}_{0}, \tilde{\mathfrak{w}}_{0}\right] \subset \mathfrak{w}_{-1}+\mathfrak{w}_{-\frac{1}{2}}+\left[\tilde{\mathfrak{w}}_{0}, \widetilde{\mathfrak{w}}_{0}\right]$. Hence, for the $k$-th derived $\mathfrak{c}^{(k)}$ we get $\mathfrak{c}^{(k)} \subset \mathfrak{w}_{-1}+\mathfrak{w}_{-\frac{1}{2}}+\widetilde{\mathfrak{w}}_{0}^{(k)}$. But $\tilde{\mathfrak{w}}_{0}$ is solvable, whence there exists $n \in N$ such that $\tilde{\mathfrak{w}}_{0}^{(n)}=0$. Then $\mathfrak{c}^{(n+2)}=0$. This finishes the proof of the theorem.

By [28], [25; Theorem 4.1] we know that adg is an algebraic Lie algebra. We use the notation of Theorem 3.12 and $[9 ; V, \S 4$, Proposition 6] to get

CoRollary 7.3. a) $\mathfrak{w}=\left[\left(\mathfrak{z}_{1}+\mathfrak{z}_{2}\right) \cap \mathfrak{w}\right]+\mathfrak{n}^{\varphi}+\mathfrak{w}_{-1}+\mathfrak{w}_{-\frac{1}{2}}$,

b) $\left(z_{1}+z_{2}\right) \cap \mathfrak{w}$ is abelian and for $X \in\left(\partial_{1}+z_{2}\right) \cap \mathfrak{w}$ is ad $X$ semisimple,

c) $\mathfrak{n}^{\varphi}+\mathfrak{w}_{-1}+\mathfrak{w}_{-\frac{1}{2}}$ is the largest nilpotent ideal of $\mathrm{g}$.

2. This paragraph is devoted to the construction of a Levi decomposition of $\mathrm{g}$. We set $\mathfrak{l}_{-1}:=\left\{X_{-1}[w] ; w \in P_{1}\right\}, \mathfrak{l}_{-\frac{1}{2}}:=\left\{X_{-\frac{1}{2}}[w] ; w \in P_{\frac{1}{2}}\right\}, \mathfrak{l}_{\frac{1}{2}}:=$ $\left\{X_{\frac{1}{2}}[w] ; w \in P_{\frac{1}{2}}\right\}$ and $\mathfrak{I}_{1}:=\left\{X_{1}[w] ; w \in P_{1}\right\}$.

We are looking for some space $\mathfrak{I}_{0}$ such that the sum of the $\mathfrak{l}_{\lambda}$ 's is an semisimple Lie algebra. We are going to describe $\mathfrak{I}_{0}$ quite explicitly. We use the Peirce decomposition $\mathfrak{U}=\bigoplus_{1 \leq i \leq j \leq q} \mathfrak{U}_{i j}$ and the spaces $\mathfrak{m}_{i j}, \mathfrak{m}_{i j}^{\varphi}, \mathfrak{a}$ and $\mathfrak{a}^{\varphi}$ which have been introduced in $\S 3$. We decompose $\mathfrak{U}_{1}=\mathfrak{U}_{11}$ into the direct sum of its simple ideals $\mathfrak{U}_{1}=\mathfrak{A}_{1}^{(1)} \oplus \cdots \oplus \mathfrak{A}_{1}^{(s)}$ and get a corresponding decomposition of $\mathfrak{m}_{11}$ and $\mathfrak{m}_{11}^{\varphi}, \mathfrak{m}_{11}=\mathfrak{m}_{11}^{(1)} \oplus \ldots \oplus \mathfrak{m}_{11}^{(s)}$ and $\mathfrak{m}_{11}^{\varphi}$ $=\mathfrak{m}_{11}^{\varphi(1)} \oplus \cdots \oplus \mathfrak{m}_{11}^{\varphi(s)}$. Without restriction we may assume $P_{1}=\mathfrak{U}_{1}^{(1)} \oplus \cdots$ $\oplus \mathfrak{U}_{1}^{(k)}$.

With this notation we put $\mathfrak{l}_{0 \mathfrak{m}}:=\mathfrak{m}_{11}^{\varphi(1)} \oplus \ldots \oplus \mathfrak{m}_{11}^{\varphi(k)}$.

LEMMA 7.4. $\left[\mathfrak{l}_{-\frac{1}{2}}, \mathfrak{l}_{\frac{1}{2}}\right] \subset \mathfrak{l}_{0 \mathfrak{m}}+\mathfrak{a}^{\varphi}$.

Proof. Let $X:=X_{-\frac{1}{2}}[w]$ and $Y:=X_{\frac{1}{2}}\left[w^{\prime}\right]$ and $(T, \hat{T}):=\frac{1}{2}[X, Y]$. Then, by (6.9), we know $T v=2 \operatorname{Re}\left(S\left(\varphi(v) w^{\prime}, w\right)\right)$ and $\hat{T} u=-\varphi(S(u, w)) w^{\prime}+$ $\varphi\left(S\left(w, w^{\prime}\right)\right) u+\varphi\left(S\left(u, w^{\prime}\right)\right) w$. From the definition of $T$, Lemma 5.1 and Corollary 5.6 we derive $T \mathfrak{A}_{1} \subset P_{1}$; further, $T \mathfrak{A}_{0}=0$ is immediate. On the other hand we may apply Theorem 3.12 ; hence there exist $\left(T_{1}, \hat{T}_{1}\right) \in \mathfrak{m}_{11}^{\varphi}$, $x_{\frac{1}{z}} \in \mathfrak{A}_{\frac{1}{2}}$ and $\left(T_{0}, \hat{T}_{0}\right) \in \bigoplus_{2 \leq i \leq j \leq q} \mathfrak{m}_{i j}^{\varphi}+\mathfrak{a}^{\varphi}$ such that $(T, \hat{T})=\left(T_{1}, \hat{T}_{1}\right)+\left(A_{c}\left(x_{\frac{1}{2}}\right)\right.$, $\left.\frac{1}{2} \varphi\left(x_{\frac{1}{2}}\right) \varphi(c)\right)+\left(T_{0}, \hat{T}_{0}\right)$ holds. The properties of $T$ mentioned above immediately imply $\left(T_{1}, \hat{T}_{1}\right) \in \mathfrak{I}_{0 \mathrm{~m}}$ and $x_{\mathrm{v}}=0$. Hence also $T_{0} \mathfrak{X}_{0}=0$. Whence $T_{0}$ $\in \mathfrak{b}$ and $\left(T_{0}, \hat{T}_{0}\right) \in \mathfrak{a}^{\varphi}$. The lemma is proved.

Denote by $\mathfrak{l}_{0 \mathrm{a}}$ the projection of $\left[\mathfrak{l}_{-\frac{1}{2}}, \mathfrak{l}_{\frac{1}{2}}\right]$ into $\mathfrak{a}^{\varphi}$. We set $\mathfrak{l}_{0}:=\mathfrak{l}_{0 \mathrm{~m}}+\mathfrak{l}_{0 \mathrm{a}}$.

In the proof of the following theorem we use the properties of $\mathfrak{a}^{\varphi}$ which we have listed in Lemma 3.14. We add that $\mathfrak{a}^{\varphi}$-annihilates $P_{1}$ and, 
by Corollary 5.9 , leaves invariant $P_{\frac{1}{2}}$.

As usual, by $p$ we denote the unit element of $P_{1}$.

TheOREM 7.5. a) $\mathfrak{l}:=\mathfrak{l}_{-1}+\mathfrak{l}_{-\frac{1}{2}}+\mathfrak{l}_{0}+\mathfrak{l}_{\frac{1}{2}}+\mathfrak{l}_{1}$ is a semisimple subalgebra of Lie Aut $D(K, S)$.

b) $\mathfrak{r}_{0 a}$ is an ideatl of $\mathfrak{a}^{\varphi}$.

c) The elements of $\mathfrak{\Upsilon}$ leave invariant the vector space $P_{1}^{C} \times P_{\frac{1}{2}}$.

d) If $X \in \mathfrak{l}$ annihilates $P_{1}^{c} \times P_{\frac{1}{2}}$ then $X=0$.

Proof. 1) In the definition of $\mathfrak{l}$ we replace $\mathfrak{l}_{0}$ by $\mathfrak{l}_{0}^{\prime}:=\mathfrak{l}_{0 \mathrm{~m}}+\mathfrak{a}^{\varphi}$. The vector space thus constructed will be denoted by $\mathfrak{l}^{\prime}$, its summands by $\mathfrak{l}_{\lambda}^{\prime}$. We claim that $\mathfrak{Y}^{\prime}$ is a subalgebra of $\mathrm{g}=$ Lie Aut $D(K, S)$ which leaves invariant $P_{1}^{C} \times P_{\frac{1}{2}}$. The second assertion is easily verified using the explicit expressions for the elements of $Y_{\lambda}^{\prime}$. To prove the first assertion it suffices to show $\left[\mathfrak{l}_{\lambda}^{\prime}, \mathfrak{Y}_{\mu}^{\prime}\right] \subset \mathfrak{Y}_{\lambda+\mu}^{\prime}$ for $\lambda, \mu \in\left\{-1,-\frac{1}{2}, 0, \frac{1}{2}, 1\right\}$. There is nothing to be proved for the pairs $(\lambda, \mu) \in\left\{(-1,-1),\left(-1,-\frac{1}{2}\right),\left(\frac{1}{2}, 1\right),(1,1)\right\}$. Next we use $\left(\mathfrak{l}_{0}^{\prime}\right)^{\sigma \oplus \rho}=\mathfrak{l}_{0}^{\prime}$, the results of $\S 6$ and Lemma 5.1 and immediately get the assertion for the pairs $(\lambda, \mu) \in\left\{(-1,0),\left(-1, \frac{1}{2}\right),(-1,1),\left(-\frac{1}{2}, 0\right),\left(-\frac{1}{2}, 1\right),\left(0, \frac{1}{2}\right)\right.$, $(0,1)\}$. To settle the cases $(\lambda, \mu) \in\left\{\left(-\frac{1}{2},-\frac{1}{2}\right),\left(\frac{1}{2}, \frac{1}{2}\right)\right\}$ we use (6.7), (6.14) and Corollary 5.6. We are left with the cases $\left[\mathfrak{l}_{0}^{\prime}, \mathfrak{l}_{0}^{\prime}\right] \subset \mathfrak{l}_{0}^{\prime}$ and $\left[\mathfrak{l}_{-\frac{1}{3}}^{\prime}, \mathfrak{l}_{\frac{1}{2}}^{\prime}\right] \subset \mathfrak{l}_{0}^{\prime}$. The first one is clear because $\mathfrak{r}_{0 \mathrm{~m}}$ and $\mathfrak{a}^{\varphi}$ commute by Lemma 3.14. The second one coincides with the assertion of Lemma 7.4.

2) We claim that $\mathfrak{l}$ is an ideal of $\mathfrak{l}^{\prime}$. We only have to prove $\left[\mathfrak{l}_{-\lambda}, \mathfrak{l}_{\lambda}\right]$ $\subset \mathfrak{r}_{0}$ for $\lambda=0, \frac{1}{2}, 1$ and $\left[\mathfrak{l}, \mathfrak{a}^{\varphi}\right] \subset \mathfrak{l}$. The case $\lambda=1$ is a consequence of (6.6) and the case $\lambda=\frac{1}{2}$ follows from the definition of $\Upsilon_{0}$. Let $\lambda=0$; then it obviously suffices to prove $\left[\mathfrak{l}_{0 a}, \mathfrak{a}^{\varphi}\right] \subset \mathfrak{l}_{0 \mathrm{a}}$. But this follows from the definition of $\mathfrak{l}_{0 a}$, the fact that $\mathfrak{a}^{\varphi}$ leaves invariant $\mathfrak{l}_{-\frac{1}{2}}$ and $\mathfrak{l}_{\frac{1}{2}}$ and that $\mathfrak{l}_{0 \mathrm{~m}}$ commutes with $\mathfrak{a}^{\varphi}$. From these remarks we also get $\left[\mathfrak{l}_{-\frac{1}{2}}+\mathfrak{l}_{0}+\mathfrak{l}_{\frac{1}{2}}, \mathfrak{a}^{\varphi}\right] \subset \mathfrak{l}$. Finally, (6.4) and (6.13) imply $\left[\mathfrak{I}_{-1}+\mathfrak{Y}_{1}, \mathfrak{a}^{\varphi}\right]=0$ and the assertion is proved.

3) The radical $\mathfrak{w}^{\prime}$ of $\mathfrak{l}^{\prime}$ is contained in the set $q$ of $X \in \mathfrak{l}_{0}^{\prime}$ which annihilate $P_{1}^{C} \times P_{\frac{1}{2}}$.

To prove this claim we first mention that the map $\sum X_{\lambda} \mapsto \sum \lambda X_{\lambda}$ is a derivation of $\mathfrak{l}^{\prime}$; therefore $\mathfrak{w}^{\prime}$ is the sum of the vector spaces $\mathfrak{w}_{\lambda}^{\prime}:=\mathfrak{w}^{\prime} \cap \mathfrak{l}_{\lambda}^{\prime}$. The proof for the fact $\mathfrak{w}_{\frac{1}{2}}^{\prime}=0$ and $\mathfrak{w}_{1}^{\prime}=0$ can be taken almost unchanged from [29; Lemma 4.2]. Hence $\left[\mathfrak{w}_{0}^{\prime}, \mathfrak{l}_{\frac{1}{2}}+\mathfrak{l}_{1}\right]=0$. We now consider the vector field $X(z, u)=\left(A_{p}(z) z, \varphi(z) \varphi(p) u\right)$ which is contained in $\mathfrak{l}_{1}^{\prime}$. As a consequence we get $(\operatorname{ad} X) \mathfrak{w}_{-\frac{1}{2}}^{\prime}=0$ and $(\operatorname{ad} X)^{2} \mathfrak{w}_{-1}^{\prime}=0$. We use (6.4), (6.6), (6.10) and Corollary 5.9 to get $\mathfrak{w}_{-\frac{1}{2}}^{\prime}=0$ and $\mathfrak{w}_{-1}^{\prime}=0$. This implies $\left[\mathfrak{w}_{0}^{\prime}, \mathfrak{l}_{-\frac{1}{2}}^{\prime}+\mathfrak{l}_{-1}^{\prime}\right]$ $=0$. By (6.4) and (6.8) the assertion follows. 
4) The Lie algebra $\mathfrak{l}$ is reductive.

From the definition of $q$ we easily derive $q \subset \mathfrak{a}^{\varphi}$. We know that $\mathfrak{a}^{\varphi}$ is reductive (see Lemma 3.14) and that $\mathfrak{w}_{0}^{\prime}$ is a solvable ideal of $\mathfrak{a}^{\varphi}$. Hence, $\mathfrak{w}_{0}^{\prime}$ is contained in the center of $\mathfrak{a}^{\varphi}$. This implies $\left[\mathfrak{l}_{0}^{\prime}, \mathfrak{l}_{0}^{\prime}\right]=0$ and $\mathfrak{w}_{0}^{\prime}$ equals the center of $\mathfrak{l}^{\prime}$. Hence, the radical $\operatorname{rad} \mathfrak{l}$ of the ideal $\mathfrak{l}$ of $\mathfrak{l}^{\prime}$ coincides with the center of $\mathfrak{l}$ and the assertion follows (see $[4 ; \S \S 5,6]$ ).

5) $\mathfrak{l} \subset[\mathfrak{l}, \mathfrak{l}]$.

To prove this claim we put $E:=\left(A(p), \frac{1}{2} \varphi(p)\right)$ and get $\left[E, \mathfrak{V}_{\lambda}\right]=\mathfrak{V}_{\lambda}$ for $\lambda \neq 0$. From (6.6) we derive $\left\{\left(A(x), \frac{1}{2} \varphi(x)\right) ; x \in P_{1}\right\} \subset[\mathfrak{l}, \mathfrak{l}]$. Whence $\mathfrak{l}_{0 \mathfrak{m}} \subset[\mathfrak{l}, \mathfrak{l}]$. By definition of $\mathfrak{l}_{0 \mathfrak{a}}$ we know $\mathfrak{l}_{0 a} \subset\left[\mathfrak{l}_{-\frac{1}{2}}, \mathfrak{l}_{\frac{1}{2}}\right]+\mathfrak{l}_{0 \mathfrak{m}}$. Hence $\mathfrak{l}_{0}=\mathfrak{l}_{0 \mathfrak{m}}+\mathfrak{l}_{0 \mathfrak{a}} \subset[\mathfrak{l}, \mathfrak{l}]$. This proves the assertion.

We are now in the position to prove the theorem. Part a) follows from 4) and 5). Part b) is contained in 2) and c) is contained in 1). It therefore suffices to prove d). Choose $X \in \mathfrak{l}$ which annihilates $P_{1}^{c} \times P_{\frac{1}{2}}$. Write $X=\sum X_{\lambda}$ where $X_{\lambda} \in \mathfrak{Y}_{\lambda}$. Now, the first components of the $X_{\lambda}$ 's are polynomials of different type; hence the first component of each $X_{\lambda}$ annihilates $P_{1}^{C} \times P_{\frac{1}{2}}$. This implies $X_{-1}=0, X_{-\frac{1}{2}}=0$ and $X_{1}=0$. Comparing the second components of the remaining $X_{2}$ 's we get along the same lines $X_{\frac{1}{2}}=0$. This means that the set $\mathfrak{x}$ of $X \in \mathfrak{l}$ which annihilate $P_{1}^{c} \times P_{\frac{1}{2}}$ is contained in $\mathfrak{r}_{0 a}$. By the results of $\S 6$ we see that $\mathfrak{x}$ commutes with $\mathfrak{r}_{-1}$ $+\mathfrak{l}_{-\frac{1}{2}}+\mathfrak{l}_{\frac{1}{2}}+\mathfrak{l}_{1}$. We also have $\left[\mathfrak{x}, \mathfrak{l}_{0 \mathrm{~m}}\right]=0$ because of $\mathfrak{x} \subset \mathfrak{l}_{00}$. Further, $\mathfrak{x}$ is an ideal of $a^{\varphi}$. Putting all together, we have shown that $\mathfrak{x}$ is an ideal of $\mathfrak{l}$. We have proved above that $\mathfrak{l}$ is semisimple; hence $\mathfrak{l}=\mathfrak{l} \oplus \tilde{\mathfrak{I}}$ where the ideal $\tilde{\mathfrak{l}}$ of $\mathfrak{l}$ equals the orthogonal complement of $\mathfrak{r}$ in $\mathfrak{l}$ with respect to the Killing form. Finally, we consider $E:=\left(A(p), \frac{1}{2} \varphi(p)\right) \in \mathfrak{l}$. From the definitions of $E$ and $\mathfrak{x}$ we easily derive $[E, \mathfrak{x}]=0$. Whence $E \in \tilde{\mathfrak{l}}$ and $[E, \mathfrak{l}] \subset \tilde{\mathfrak{l}}$. In 5$)$ we have shown that $[E, \mathfrak{l}]=\mathfrak{l}_{-1}+\mathfrak{l}_{-\frac{1}{2}}+\mathfrak{l}_{\frac{1}{2}}+\mathfrak{l}_{1}$ generates the Lie algebra $\mathfrak{l}$. This implies $\mathfrak{x}=0$ and the theorem is proved.

CoRollary 7.6. a) $\mathfrak{l}_{0}$ is generated by $\left[\mathfrak{l}_{-\frac{1}{2}}, \mathfrak{l}_{-\frac{1}{2}}\right]+\left[\mathfrak{l}_{-1}, \mathfrak{l}_{1}\right]$.

b) Let $(T, \hat{T}) \in \mathfrak{I}_{0 \mathrm{a}}$ and $v \in V$ satisfying $p v=0$, then $T v=0$.

Proof. a) is a consequence of 5) from the proof of Theorem 7.5 and b) is contained in the proof of Lemma 7.4.

Next we describe $\mathfrak{a}^{\varphi}$ more precisely. We set $t_{\mathfrak{a}}:=\left\{X \in \mathfrak{a}^{\varphi} ; X\left(P_{1}^{C} \times P_{\frac{1}{2}}\right)\right.$ $=0\}$. From d) of Theorem 7.5 we get $t_{a} \cap \mathfrak{r}_{0 a}=0$.

CoRollary 7.7. The Lie algebra $\mathfrak{a}^{\varphi}$ is the direct sum of its ideals $t_{a}$ and $\mathfrak{l}_{0 a}, \mathfrak{a}^{\varphi}=\mathfrak{t}_{\mathfrak{a}} \oplus \mathfrak{I}_{0 a}$. 
The Lie algebra $t_{a}$ is reductive and its center is contained in the radical of $\mathfrak{g}(K, S), \mathrm{t}_{\mathfrak{a}}=\left[\mathrm{t}_{\mathfrak{a}}, \mathrm{t}_{\mathfrak{a}}\right] \oplus \mathfrak{w}_{0} \cap \mathfrak{a}^{\varphi}$.

Proof. It is clear that $t_{a}$ is an ideal of $a^{\varphi}$ and, as carried out in the

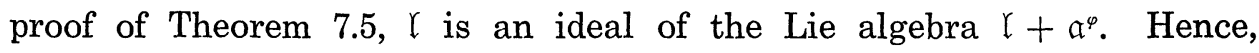
ad $X$ is a derivation of the semisimple Lie algebra $\mathfrak{l}$ for all $X \in a^{\varphi}$. Therefore, there exists $Y \in \mathfrak{l}$ such that ad $(X-Y) \mathfrak{l}=0$ holds. We apply ad $(X-Y)$ to $\left(A(p), \frac{1}{2} \varphi(p)\right) \in \mathfrak{l}$ and get $Y \in \mathfrak{l}_{0}$. We know that $Y=Y_{\mathrm{m}}+Y_{a}$ where $Y_{\mathrm{m}} \in \mathfrak{l}_{0 \mathrm{~m}}$ and $Y_{\mathrm{a}} \in \mathfrak{l}_{0 \mathrm{a}}$ and derive from ad $(X-Y) \mathfrak{l}=0$ easily $Y=Y_{\mathrm{a}}$. Hence $X-Y \in \mathfrak{a}^{\varphi}$. Finally, ad $(X-Y) \mathfrak{l}_{-\frac{1}{2}}=0$ shows that $X-Y$ annihilates $P_{1}^{c} \times P_{\frac{1}{1}}$ and thus proves the first assertion. Let now $X$ lie in the center $\mathfrak{o}$ of $t_{a}$. Then $X$ is contained in the center of $\mathfrak{a}^{\varphi}$. By Theorem 3.12 we thus get $X \in \operatorname{rad} g_{0}$. But, by definition of $t_{a}$, we also have $T^{\sigma} P_{1}=0$ and $\hat{T}^{\rho} P_{\frac{1}{2}}=0$ where $X=(T, \hat{T})$; hence, by Theorem 7.2 , we get $X \in \mathfrak{w}_{0}$. Finally, the ideal $t_{a}$ of the reductive algebra $a^{\varphi}$ is reductive again, whence $t_{a}=\left[t_{a}, t_{a}\right]+\mathfrak{b}$. But we have shown above $\mathfrak{o} \subset \mathfrak{w}_{0} \cap t_{a}$ and the assertion follows.

We are now in the position to provide a Levi decomposition where the semisimple summand has especially nice properties. Let $\mathfrak{h}_{i j}$ (resp. $\mathfrak{h}_{11}^{(r)}$ resp. $\mathfrak{h}_{\mathfrak{b}}$ ) denote the semisimple part of the reductive Lie algebra $\mathfrak{m}_{j j}^{\varphi}$ (resp. $\mathfrak{m}_{11}^{\varphi(r)}$ resp. $\left.\mathfrak{t}_{\mathrm{a}}\right)$

We set $\mathfrak{h}:=\bigoplus_{r=k+1}^{s} \mathfrak{h}_{11}^{(r)}+\bigoplus_{j=2}^{q} \mathfrak{h}_{j j}+\mathfrak{h}_{a}$.

THEOREM 7.8. a) The sum $\mathfrak{l}+\mathfrak{h}$ of Lie algebras is direct.

b) $\mathfrak{r}+\mathfrak{h}$ is a maximal semisimple subalgebra of $\mathfrak{g}(K, S)$.

Proof. It is clear that the sum of the vector spaces $\mathfrak{l}$ and $\mathfrak{h}$ is direct. The Lie algebras $\mathfrak{m}_{j j}$ and $\mathfrak{m}_{r r}$ commute as do $\mathfrak{m}_{11}^{\varphi(j)}$ and $\mathfrak{m}_{11}^{\varphi(r)}$ if $j \neq r$. Further, $\left[\mathfrak{m}_{r r}^{\varphi}, \mathfrak{a}^{\varphi}\right]=0$ for $1 \leq r \leq q$ and $\left[\mathfrak{l}_{0 a}, \mathfrak{t}_{a}\right]=0$. To finish the proof of a) it now suffices to note that, by the results of $\S 6$, we have $\left[\mathfrak{h}, \mathfrak{l}_{-1}+\right.$ $\left.\mathfrak{l}_{-\frac{1}{2}}+\mathfrak{l}_{\frac{1}{2}}+\mathfrak{r}_{1}\right]=0$. To prove b) it is enough to show $\mathfrak{g}=\mathfrak{g}(K, S)=\mathfrak{l}+\mathfrak{h}$ $+\mathfrak{w}$. We use the definition of $\mathfrak{l}$ and Theorem 7.1 and see that we only have to prove $\mathfrak{g}_{0} \subset \mathfrak{l}_{0}+\mathfrak{h}+\mathfrak{w}_{0}$. We apply Theorem 7.2 and get that the centers of $\mathfrak{m}_{11}^{\varphi(r)}, r>k$, and $\mathfrak{m}_{j j}^{\varphi}, j \geq 2$, are contained in $\mathfrak{w}_{0}$. Hence,-see Theorem 3.12-it suffices to show $\mathfrak{a}^{\varphi} \subset \mathfrak{l}_{0 a}+\mathfrak{h}_{\mathfrak{a}}+\mathfrak{w}_{0} \cap \mathfrak{a}^{\varphi}$. But, by Corollary 7.7, we know $\mathfrak{h}_{\mathfrak{a}}+\mathfrak{w}_{0} \cap \mathfrak{a}^{\varphi}=\mathfrak{t}_{\mathfrak{a}}$. The theorem is proved.

3. In [38] it was shown that $g=g(K, S)$ contains a (possibly trivial) semisimple subalgebra which is canonically isomorphic to the Lie algebra of infinitesimal automorphisms of a symmetric Siegel domain. In this 
paragraph we construct a symmetric Siegel domain $D\left(K^{\prime}, S^{\prime}\right) \subset D(K, S)$ and prove that the restriction of the Lie algebra $\mathfrak{l}$ (which has been constructed in the last section) to $D\left(K^{\prime}, S^{\prime}\right)$ coincides with Lie Aut $D\left(K^{\prime}, S^{\prime}\right)$. This makes concrete the rather abstract construction of [38].

We recall that a Siegel domain is called symmetric if its Lie algebra of infinitesimal automorphisms is semisimple [58], [60], [61].

We define $V^{\prime}:=P_{1}, U^{\prime}:=P_{\frac{1}{2}}$ and $S^{\prime}:=\left.S\right|_{U^{\prime} \times U^{\prime}}$. Further, by $K^{\prime}$ we denote the projection of $K$ into $V^{\prime}$. Then, by Corollary $6.6, S^{\prime}$ is a $K^{\prime}$ hermitian form. We therefore may consider the Siegel domain $D\left(K^{\prime}, S^{\prime}\right)$.

Theorem 7.9. The Siegel domain $D\left(K^{\prime}, S^{\prime}\right)$ is homogeneous and $\mathfrak{l}$ is canonically isomorphic to Lie Aut $D\left(K^{\prime}, S^{\prime}\right)$. The Siegel domain $D\left(K^{\prime}, S^{\prime}\right)$ is symmetric.

Proof. 1) We identify $\mathfrak{l}$ with its restriction to $P_{1}^{c} \times P_{\frac{1}{2}}$. This can be done because of part d) of Theorem 7.5. If $P_{1}$ decomposes into the sum $P_{1}=\mathfrak{Q}_{1}^{(1)} \oplus \cdots \oplus \mathfrak{N}_{1}^{(k)}$, then also $\mathfrak{l}$ decomposes. We therefore may assume that $P_{1}$ is simple.

2) We compute $\eta_{K^{\prime} s^{\prime}}, \rho^{\prime}, \varphi^{\prime}$ for $D\left(K^{\prime}, S^{\prime}\right)$. Put

$$
\eta^{\prime}(x):=\left[\iota_{K^{\prime}}(x)\right]^{2}\left[\left.\operatorname{det} \varphi(x)\right|_{P_{\frac{1}{2}}}\right]^{-1}, \quad x \in K^{\prime},
$$

and $W:=\exp t A(y), \hat{W}:=\exp t \varphi(y), y \in P_{1}$; then

$$
\eta^{\prime}(W x)=[\operatorname{det} W]^{-2}[\operatorname{det} \hat{W}]^{-2} \eta^{\prime}(x)
$$

follows. Hence, $\eta_{K^{\prime} S^{\prime}}$ and $\eta^{\prime}$ only differ by a positive constant factor. Further, it is clear that $\omega(x):=\left.\operatorname{det} \varphi(x)\right|_{P_{\frac{1}{2}}}$ is a "multiplicative polynomial" in the sense of $[5 ; \mathrm{II}, \S 3]$ and therefore, by [5; III, $\S 6]$ a power of $\iota_{K^{\prime}}(x)$. This implies that there exist $\alpha>0, \beta>0$ such that $\eta_{K^{\prime} s^{\prime}}(x)=\alpha\left[\iota_{K^{\prime}}(x)\right]^{\beta}$ holds for all $x \in K^{\prime}$. We now apply the results of [31; VI] on domains of positivity and formally real Jordan algebras and see that the algebra which is defined for $\eta_{K^{\prime} S^{\prime}}$ and the unit $p$ of $P_{1}$ according $\S 3$ coincides with the ideal $P_{1}$ of $\mathfrak{A}_{1}$. From this and the fact that $P_{1}$ is simple we get that the bilinear form $\sigma^{\prime}$ which is defined for $\eta_{K^{\prime} s^{\prime}}$ and $p$ and the bilinear form $\left.\sigma\right|_{P_{1} \times P_{1}}$ only differ by some positive factor $\delta$ because both bilinear forms are associative. We use the definition of $\rho^{\prime}$ and see $\rho^{\prime}=\left.\delta \rho\right|_{P_{\frac{1}{2}} \times P_{\frac{1}{2}}}$. Whence $\varphi^{\prime}(x)=\left.\varphi(x)\right|_{P_{\frac{1}{2}}}$ for all $x \in P_{1}$.

3) We set $\mathfrak{g}^{\prime}:=$ Lie Aut $D\left(K^{\prime}, S\right), \mathfrak{g}^{\prime}=\mathfrak{g}_{-1}^{\prime}+\mathfrak{g}_{-\frac{1}{2}}^{\prime}+\mathfrak{g}_{0}^{\prime}+\mathfrak{g}_{\frac{1}{2}}^{\prime}+\mathfrak{g}_{1}^{\prime}$. From 2) and Theorems 4.1 and 5.4 we get $\mathfrak{l}_{-1}=\mathfrak{g}_{-1}^{\prime}, \mathfrak{l}_{-\frac{1}{2}}=\mathfrak{g}_{-\frac{1}{2}}^{\prime}, \mathfrak{l}_{\frac{1}{2}}=\mathfrak{g}_{\frac{1}{2}}^{\prime}$ and $\mathfrak{l}_{1}=$ $\mathfrak{g}_{1}^{\prime}$. By Theorem 1.6 we thus have $\mathfrak{w}_{-1}^{\prime}=0$ and $\mathfrak{w}_{-\frac{1}{2}}^{\prime}=0$. But now (6.4) 
and (6.8) imply $\mathfrak{w}_{0}^{\prime}=0$ whence $\mathfrak{g}^{\prime}$ is semisimple. We therefore only have to prove $\mathfrak{l}_{0}=\mathfrak{g}_{0}^{\prime}$. We now apply Corollary 7.6 to $\mathfrak{l}$ and to $\mathfrak{g}^{\prime}$. But $\mathfrak{l}_{\lambda}=\mathfrak{g}_{\lambda}^{\prime}$ for $\lambda \neq 0$ and $\mathfrak{r}_{0}=\mathfrak{g}_{0}^{\prime}$ follows. The theorem is proved.

Remark 7.10. Put $E_{s}:=\left(A(p), \frac{1}{2} \varphi(p)\right)$; then, by the results of this paper, most of the results of $[38 ; \S \S 2,3]$ are immediate consequences.

\section{LITERATURVERZEICHNIS}

[1] Aronszain, N., Theory of reproducing kernels, Trans. Amer. Math. Soc., 68 (1950), 337-404.

[2] Atiyah, M., Bott, R. and Shapiro, A., Clifford modules, Topology, 3 (1964), 3-38.

[ 3 ] Bourbaki, N., Algebre, chap. 8, Hermann, Paris, 1958.

[4] - Groupes et Algebres de Lie, chap. I, Hermann, 1971.

[ 5 ] Braun, H. and Koecher, M., Jordan-Algebren, Springer, Berlin-Heidelberg-New York, 1966.

[6] Cartan, E., Domaines bornés dans l'éspace de $n$ variables complexes, Abh. Math. Sem. Hamburg, 11 (1935), 116-162.

[ 7 ] Cartan, H., Sur les groupes de transformations analytiques, Actualités Sc. et Indus. Hermann, Paris, 1935.

[ 8 ] Chevalley, C., The Algebraic Theory of Spinors, Columbia Univ. Press, Morningside Heights, New York, 1954.

[9] —-, Théorie des groupes de Lie, Hermann, Paris, 1968.

[10] Dorfmeister, J., Eine Theorie der homogenen, regulären Kegel, Dissertation, Univ. Münster, 1974.

[11] —- Zur Konstruktion homogener Kegel, Math. Ann., 216 (1975), 79-96.

[12] — Infinitesimal automorphisms of homogeneous Siegel domains, preprint, 1975.

[13] —- Homogene Siegel-Gebiete, Habilitationsschrift, Münster, 1978.

[14] —, Inductive construction of homogeneous cones, Trans. Amer. Math. Soc., 252 (1979), 321-349.

[15] — Algebraic description of homogeneousc ones, Trans. Amer. Math. Soc., 255 (1979), 61-89.

[16] Dorfmeister, J. and Koecher, M., Relative Invarianten und nichtassoziative Algebren, Math. Ann., 228 (1977), 147-186.

[17] —-, Reguläre Kegel, Jber. Deutsch. Math.- Verein., 81 (1979), 109-151.

[18] Dorfmeister, J., Peirce-Zerlegungen und Jordan-Strukturen zu homogenen Kegeln, Math. Z., 169 (1979), 179-194.

[19] Gindikin, S. G., Analysis in homogeneous domains, Russian Math. Surveys, 19 (1964), 1-89.

[20] Gindikin, S. G., I. I. Piatetsky-Shapiro and E. B. Vinborg, On the classification and canonical realization of homogeneous bounded domains, Trans. Moscow Math. Soc., 12 (1963), 404-437.

[21] —, Homogeneous Kähler manifolds, in Geometry of Homogeneous Bounded Domains, C.I.M.E. Edizioni Cremonese, Roma, 1967.

[22] Helgason, S., Differential Geometry and Symmetric Spaces, Academic Press, New York and London, 1962.

[23] Helwig, K.-H., Halbeinfache reelle Jordan-Algebren, Math. Z., $10[9$ (1969), 1-28.

[24] Jacobson, N., Structure and Representations of Jordan algebras, Amer. Math. Soc. Coll. Publ., Providence, 1968. 
[25] Kaneyuki, S., On the automorphism groups of homogeneous bounded domains, J. Fac. Sci. Univ. Tokyo, 14 (1967), 89-130.

[26] - Homogeneous Bounded Domains and Siegel Domains, Lecture Notes in Mathematics 241, Springer, Berlin-Heidelberg-New York, 1971.

[27] Kaneyuki, S. and Tsuji, T., Classification of homogeneous bounded domains of lower dimension, Nagoya Math. J., 53 (1974), 1-46.

[28] Kaup, W., Einige Bemerkungen über polynomiale Vektorfelder, Jordanalgebren und die Automorphismen von Siegelschen Gebieten, Math. Ann., 204 (1973), 131144.

[29] Kaup, W., Matsushima, Y. and Ochiai, T., On the automorphisms and equivalences of generalized Siegel domains, Amer. J. Math., 92 (1970), 475-497.

[30] Kobayashi, Hyperbolic Manifolds and Holomorphic Mappings, Pur and Applied Mathematics, Marcel Dekker, New York, 1970.

[31] Koecher, M., Jordan-Algebras and their Applications, Lecture Notes, Univ. Minnesota, 1962.

[32] - An, Elementary Approach to Bounded Symmetric Domains, Rice Univ., Houston, 1969.

[33] — Eine Konstruktion von Jordan-Algebren, manuscripta math., 23 (1978), 387425.

[34] Loos, O., Jordan triple systems, $R$-spaces, and bounded symmetric domains, Bull. Amer. Math. Soc., 77 (1971), 558-561.

[35] — B Bounded symmetric domains and Jordan pairs, Math. Lectures, Univ. California Irvine, 1977.

[36] Murakami, S., On Automorphisms of Siegel Domains, Lecture Notes in Mathematics 286, Springer, Berlin-Heidelberg-New York, 1971.

[37] Nakajima, K., Some studies on Siegel domains, J. Math. Soc. Japan, 27 (1975), 54-75.

[38] — Symmetric spaces associated with Siegel domains, J. Math. Kyoto Univ., 15 (1975) , 303-349.

[39] - On realization of Siegel domains of the second kind as those of the third kind, J. Math. Kyoto Univ., 16 (1976), 143-166.

[40] Narasimhan, R., Several Complex Variables, The Univ. of Chicago Press, Chicago and London, 1971.

[41] Piatetsky-Shapiro, I., On a problem proposed by E. Cartan, Dokl. Akad. Nauk. SSSR, 113 (1957), 980-983.

[42] - Géometrie des domaines classiques et théorie des fonctions automorphes, Dunod, Paris, 1966.

[43] - The geometry and classification of bounded homogeneous domains, Russian Math. Surveys, 20 (1966), 1-48.

[44] Rothaus, O., Automorphisms of Siegel domains, Trans. Amer. Math. Soc., 162 (1971), 351-382.

[45] —-, Automorphisms of Siegel domains, Amer. J. Math., 101 (1979), 1167-1179.

[46] Satake, I., On classification of quasi-symmetric domains, Nagoya Math. J., 62 (1976), 1-12.

[47] —- Algebraic structures of symmetric domains, Iwanami Shoten and Princeton Univ. Press, 1980.

[48] — Infinitesimal Automorphisms of Symmetric Siegel Domains, unpublished manuscript, will be contained in [47].

[49] Sudo, M., On infinitesimal automorphisms of some non-degenerate Siegel domains, J. Fac. Sci. Univ. Tokyo, 22 (1975), 429-436.

[50] —, A remark on Siegel domains of second kind, J. Fac. Sci. Univ. Tokyo, 22 
(1975), 199-203.

[51] Sudo, M., On infinitesimal automorphisms of Siegel domains over classical cones, preprint.

[52] Takeuchi, M., Homogeneous Siegel Domains, Pub. Study Group Geometry, No. 7, Tokyo 1973.

[53] Takeuchi, On symmetric Siegel domains, Nagoya Math. J., 59 (1975), 9-44.

[54] Tanaka, N., On infinitesimal automorphisms of Siegel domains, J. Math. Soc. Japan, 22 (1970), 180-212.

[55] Tsuji, T., Classification of homogeneous Siegel domains of type II of dimensions 9 and 10, Proc. Japan Acad., 49 (1973), 394-396.

[56] - On infinitesimal automorphisms and homogeneous Siegel domains over circular cones, Proc. Japan Acad., 49 (1973), 33-80.

[57] — Siegel domains over self-dual cones and their automorphisms, Nagoya Math. J., 55 (1974) , 33-80.

[58] Vey, J., Sur la division des domaines de Siegel, Ann. Sci. Ec. Norm. Sup., 3 (1970), 479-506.

[59] Vinberg, E., B., The theory of convex homogeneous cones, Trans .Moscow Math. Soc., 12 (1963), 340-403.

[60] Borel, A., Kahlerian coset spaces of semi-simple Lie groups, Proc. Nat. Akad. Sci. U.S.A., 40 (1954), 1147-1151.

[61] Koszul, Sur la forme hermitienne canonique des espaces homogenes complexes, Canad. J. Math., 7 (1955), 562-576.

[62] Dorfmeister, J., Quasisymmetric Siegel domains and the automorphisms of homogeneous Siegel domains, Amer. J. Math., 102 (1980), 537-563.

Westfälische Wilhelms-Universität 
